\title{
COHOMOLOGICALLY SYMPLECTIC SPACES: TORAL ACTIONS AND THE GOTTLIEB GROUP
}

\author{
GREGORY LUPTON AND JOHN OPREA
}

\begin{abstract}
Aspects of symplectic geometry are explored from a homotopical viewpoint. In particular, the question of whether or not a given toral action is Hamiltonian is shown to be independent of geometry. Rather, a new homotopical obstruction is described which detects when an action is Hamiltonian. This new entity, the $\lambda_{\hat{\alpha}}$-invariant, allows many results of symplectic geometry to be generalized to manifolds which are only cohomologically symplectic in the sense that there is a degree 2 cohomology class which cups to a top class. Furthermore, new results in symplectic geometry also arise from this homotopical approach.
\end{abstract}

\section{INTRODUCTION}

Because Hamiltonian symmetries of a mechanical system lead to a reduction of order (cf. [A-M, Ol]), it is important to study Lie group actions on the types of manifolds arising in mechanics, the symplectic (and Poisson) manifolds. In particular, much work has been done in recent years on circle (and, more generally, torus) actions on closed symplectic manifolds (cf., for example, $\left[\mathrm{Au}_{1}\right]$ (and the references contained therein), $\left[\mathrm{McD}_{2}, \mathrm{On}_{1}, \mathrm{On}_{2}, \mathrm{Gi}_{1}\right.$, A-B]). Because the reduction of order mentioned above requires Hamiltonian symmetries, one focus of the investigation of symplectic actions has been the determination of 'Hamiltonianness' and its geometric consequences. Often (as we shall see below), such results include algebraic topological hypotheses which are used in very geometric arguments. The purpose of this paper is to point out an algebraic topological foundation which underlies many of these results and which allows for generalization to manifolds which are not quite symplectic and arbitrary actions on them. In this way, at least for the study of actions on symplectic manifolds, we hope to clarify the relationship between topology and geometry.

Recall that a compact symplectic manifold $\left(M^{2 n}, \omega\right)$ is a closed manifold $M$ together with a closed nondegenerate 2-form $\omega$. The nondegeneracy condition is equivalent to saying that $\omega^{n}$ is a volume form on $M$ (and therefore is a generator for the top cohomology over $\mathbb{R}$ ). Any Kähler manifold can be viewed as a symplectic manifold, by taking the Kähler form as a symplectic form. However, there are many examples of non-Kählerian, symplectic manifolds [Th], [C-F-G], $\left[\mathrm{McD}_{1}\right]$ The symplectic manifold $M$ is said to be of

Received by the editors February 17, 1994.

1991 Mathematics Subject Classification. Primary 57R19, 57S25, 55P99, 58F05. 
Lefschetz type if multiplication by the cohomology class $\omega^{n-1}$ is an isomorphism from $H^{1}(M)$ to $H^{2 n-1}(M)$. All compact Kähler manifolds satisfy this property. Indeed, a compact Kähler manifold has the hard Lefschetz property, whereby multiplication by the cohomology class $\omega^{n-r}$ is an isomorphism from $H^{r}(M)$ to $H^{2 n-r}(M)$ for each $r$.

The hypothesis of Lefschetz type, rather than the more restrictive one of Kählerian, is common in what follows. We mention here a way to obtain nonKählerian, symplectic manifolds of Lefschetz type: Let $\left(X^{2 m}, \omega_{X}\right)$ be a Kähler manifold and let $\left(Y^{2 n}, \omega_{Y}\right)$ be a simply-connected symplectic manifold. Their product $X \times Y$ is a $(2 m+2 n)$-dimensional symplectic manifold, with symplectic class $\omega_{X}+\omega_{Y}$. One easily checks that it is of Lefschetz type. Now, it is wellknown, and easy to show, that if a manifold $M$ admits a Kähler structure - more generally, has cohomology with the hard Lefschetz property - then all odd Betti numbers $b_{2 i+1}(M)$ are even. So suppose in addition that the symplectic manifold $Y$ has some odd Betti number that is odd. Then so too does the product $X \times Y$. For if $b_{2 i+1}(Y)$ is the first odd Betti number which is odd, then

$$
\begin{aligned}
b_{2 i+1}(X \times Y)= & b_{2 i+1}(X)+b_{2 i}(X) b_{1}(Y)+b_{2 i-1}(X) b_{2}(Y) \\
& +\cdots+b_{1}(X) b_{2 i}(Y)+b_{2 i+1}(Y) .
\end{aligned}
$$

Since $X$ is Kähler, each $b_{2 j+1}(X)$ is even, and by assumption each $b_{2 j+1}(Y)$ for $j<i$ is even. Hence each term in the right-hand sum except the last, which is odd, is even and so $b_{2 i+1}(X \times Y)$ is odd. It follows that $X \times Y$ cannot admit a Kähler structure. Furthermore, if $X$ is a Kähler manifold and if $Y$ is a symplectic manifold of Lefschetz type, then $X \times Y$ is again of Lefschetz type. As previously, if $Y$ has an odd Betti number that is odd, so does the product, which therefore cannot admit a Kähler structure. Starting with McDuff's examples $\left[\mathrm{McD}_{1}\right]$ of simply-connected, symplectic manifolds that have an odd Betti number which is odd, one obtains in this way many examples of symplectic manifolds of Lefschetz type that cannot admit a Kähler structure.

We review some of the standard terminology relating to Hamiltonian $S^{1}$ actions: The circle $S^{1}$ acts symplectically on the symplectic manifold $\left(M^{2 n}, \omega\right)$ if each $g \in S^{1}$ satisfies $g^{*} \omega=\omega$ as forms. We denote the action by $A$ : $S^{1} \times M \rightarrow M$ and the associated orbit map at $m$ by $a_{m}(g)=A(g, m)$ (or simply $a$ when no confusion can arise). The orbit map induces a map of tangent spaces $T_{e} a: T_{e} S^{1} \rightarrow T_{m} M$ for any given $m$, so by fixing a unit vector $X$ in $T_{e} S^{1} \cong \mathbb{R}$ and mapping by $T_{e} a$ we obtain a fundamental vector field $\underline{X}$ on $M$. Recall that the interior multiplication operator $i_{\underline{X}}$ is defined on a $k$-form $\theta$ by $i(\underline{X}) \theta\left(Y_{1}, \ldots, Y_{k-1}\right)=\theta\left(\underline{X}, Y_{1}, \ldots, Y_{k-1}\right)$. The operator $i_{\underline{X}}$ is a derivation on the commutative differential graded algebra of DeRham forms and it satisfies

$$
L_{\underline{X}}=i(\underline{X}) d+d i(\underline{X}),
$$

where $L_{\underline{X}}$ is the Lie derivative and $d$ is exterior differentiation. The definition of $L_{\underline{X}}$ and the $S^{1}$-invariance of the closed form $\omega$ imply that

$$
0=L_{\underline{X}}(\omega)=d i(\underline{X}) \omega .
$$

Hence, $i(\underline{X}) \omega$ is a closed 1-form. The $S^{1}$-action is said to be Hamiltonian if $i(\underline{X}) \omega$ is exact; that is, if there is a smooth function $\mu: M \rightarrow \mathbb{R}$ with 
$d \mu=i(\underline{X}) \omega$. This description may, of course, be generalized to torus actions as well. In this paper we consider a more general cohomological (as opposed to geometrical) framework.

Definition. A manifold $M^{2 n}$ is cohomologically-symplectic (or c-symplectic) if there is a class $\omega \in H^{2}(M ; \mathbb{Q})$ such that $\omega^{n}$ is a top class for $M$. As above, a c-symplectic manifold has Lefschetz type if multiplication by $\omega^{n-1}$ is an isomorphism from $H^{1}(M ; \mathbb{Q})$ to $H^{2 n-1}(M ; \mathbb{Q})$.

Since $S^{1}$ is path-connected, an $S^{1}$-action has $g^{*}=\mathrm{id}_{H^{*}(M)}$. Hence an $S^{1-}$ action on a c-symplectic manifold $M$ preserves the class $\omega$. Thus, in our results below (e.g., Theorems 3.8, 4.16 and 5.5), we do away with the requirement that the action be symplectic. A fundamental question in symplectic geometry is whether, for a closed manifold, c-symplectic and a given almost complex structure imply symplectic. For nilmanifolds $\left[B-G_{1}\right]$, certain solvmanifolds $\left[B-G_{2}\right]$ and orientable $T^{2}$-bundles over $T^{2}[\mathrm{Ge}$ the answer to this question is yes. Indeed, in these cases there is no distinction between c-symplectic and symplectic. In general however, the answer is unknown. Bob Gompf has pointed out to us that even $\mathbb{C} P^{2} \# \mathbb{C} P^{2}$ is an example of a c-symplectic manifold which is not symplectic since it has no almost complex structure. (See [ $\mathrm{Au}_{2}$, Proposition 1.3.1] for a general result applicable to any connected sum of symplectic 4-manifolds.) Finally, it is relatively easy to construct Sullivan minimal models which are c-symplectic [L-O], many of which are known (by rational surgery) to be realized by smooth manifolds. No good approach to ascertaining whether these manifolds are actually symplectic appears to be forthcoming, however. These questions and examples point up the utility of our topological approach. Furthermore, as in Theorems 3.11, 4.16, 5.9, and 5.11, and Corollaries 5.15 and 5.17, our methods have something new to say about symplectic geometry as well.

In $\S 2$ we describe a derivation of degree -1 on $H^{*}(M ; \mathbb{Q})$, denoted by $\lambda_{\hat{\alpha}}$, and associated to an element $\hat{\alpha} \in \pi_{1}\left(M^{M}, 1_{M}\right)$, for any space $M$. In particular, any $S^{1}$-action on the space $M$ provides such an $\hat{\alpha}$, hence a corresponding $\lambda_{\hat{\alpha}}$. In case $(M, \omega)$ is a symplectic manifold and the $S^{1}$-action is symplectic, we show in Theorem 2.3 that $\lambda_{\hat{\alpha}}(\omega)=[i(\underline{X}) \omega]$. Thus, even when $[i(\underline{X}) \omega]$ is not defined, we may call an action cohomologically Hamiltonian, or c-Hamiltonian, if $\lambda_{\hat{\alpha}}(\omega)=0$.

In case a c-symplectic manifold $M$ has Lefschetz type, Theorem 3.8 identifies c-Hamiltonian actions as those actions having fixed points or, equivalently, as those actions whose orbit maps are trivial on homology. This then is a generalization of the following result:

Theorem $\mathrm{A}$ (cf. [ $\left.\mathrm{Fr}, \mathrm{On}_{1}\right]$ ). For a symplectic $S^{1}$-action on a symplectic manifold of Lefschetz type, the following are equivalent:

(i) The action is Hamiltonian.

(ii) The action has fixed points.

(iii) The orbit map induces zero on homology.

Further, as in the next result, our approach allows us to apply results of Gottlieb in a very direct way to symplectic geometry itself:

Theorem 3.11. Suppose the first Chern class of a symplectic manifold $(M, \omega)$ is a nonzero multiple of $\omega$. Then any symplectic $S^{1}$-action on $M$ is Hamiltonian. 
Remark. After several versions of the present work had been completed, we came upon the paper $\left[\mathrm{McD}_{3}\right]$ of Dusa McDuff in which she uses Gottlieb's results to prove the vanishing of the Calabi invariant under the Chern class assumption above. Indeed, McDuff's beautiful paper is, in some sense, a direct symplectic ancestor to the present work. Below, following Theorem 3.11, we shall discuss the relationship of the $\lambda_{\hat{\alpha}}$-invariant with the Calabi invariant [Cal] and results of $\mathrm{McDuff}\left[\mathrm{McD}_{3}\right.$ ], Banyaga [Ban] and Ginzburg [Gi ${ }_{2}$ ].

In $\S 4$ we study the situation where $\omega \in H^{2}(M ; \mathbb{Q})$, thought of as an element in $\operatorname{Hom}\left(H_{2}(M ; \mathbb{Z}), \mathbb{Q}\right)$, vanishes on the image of the Hurewicz homomorphism. We denote this by $\left.\omega\right|_{\pi_{2} M}=0$. We show that this hypothesis reduces many questions about the c-symplectic structure of $M$ to the fundamental group level. In case $M$ is a $K(\pi, 1)$ of Lefschetz type, we can even obtain information about the fundamental group itself (compare Corollary 4.6). In particular, this viewpoint has the immediate consequence

Theorem B (cf. [B- $\left.\mathrm{G}_{1}, \mathrm{McD}_{2}, \mathrm{~L}-\mathrm{O}\right]$ ). A symplectic nilmanifold of Lefschetz type is diffeomorphic to a torus.

Also, our approach to $S^{1}$-actions on c-symplectic manifolds with $\left.\omega\right|_{\pi_{2} M}=0$ points out the powerful cohomological influence of the fundamental group on these actions. In particular, by combining Theorems 4.12 and 4.15 , we obtain the following result:

Theorem. Suppose $(M, \omega)$ is a c-symplectic manifold and that $\left.\omega\right|_{\pi_{2} M}=0$. If $\alpha: S^{1} \rightarrow M$ is the orbit map of a nontrivial $S^{1}$-action on $M$, then $\alpha$ is of infinite order in $\pi_{1} M$.

As a special case of this proposition, we obtain the following result:

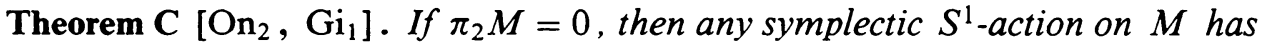
no fixed points.

Finally, $\S 5$ extends our results to sets of elements $\left\{\hat{\alpha}_{1}, \ldots, \hat{\alpha}_{k}\right\}$ in $\pi_{1}\left(M^{M}, 1_{M}\right)$. These arise naturally in conjunction with toral actions. The extensions of our results for circle actions include generalizations of Ginzburg's notion of a cohomologically free action and the following result of his:

Theorem $\mathbf{D}\left[\mathrm{Gi}_{1}\right]$. If $T^{k}$ acts symplectically on a symplectic manifold, then there is a decomposition $T^{k}=T^{r} \times T^{k-r}$ with the restriction of the action cohomologically free on $T^{r}$ and Hamiltonian on $T^{k-r}$.

Furthermore, in the Lefschetz type case, we identify cohomologically free actions with homologically injective ones (Theorem 5.9). The latter are actions of $T^{k}$ for which the homomorphism $H_{1}\left(T^{k} ; \mathbb{Z}\right) \rightarrow H_{1}(M ; \mathbb{Z})$ induced by the orbit map is injective. We proceed to show the following results:

Theorem 5.11. Suppose $(M, \omega)$ has Lefschetz type. Then $T^{k}$ acts cohomologically freely on $M$ if and only if all isotropy groups are finite.

Suppose a torus $T^{k}$ acts with finite isotropy on $(M, \omega)$ of Lefschetz type. By the discussion above, the action is homologically injective, so $\operatorname{dim} H^{*}(M) \geq$ $2^{k}$. This argument then proves the conjecture of Halperin [Ha] in the case of manifolds of Lefschetz type (cf. Theorem 5.16). We also have 
Corollary 5.13. If $(M, \omega)$ is c-symplectic with $\left.\omega\right|_{\pi_{2} M}=0$, then any $T^{k}$-action on $M$ is cohomologically free. If $M$ also has Lefschetz type, then any $T^{k}$-action is homologically injective.

Homologically injective actions have been studied extensively and we apply some of this knowledge to the cohomologically free situation. This produces cohomological and topological structure results such as Corollaries 5.15 and 5.17 .

Throughout this paper we shall only consider closed manifolds. Further, unless specifically mentioned or denoted otherwise, all homology and cohomology shall have coefficients in $\mathbb{Q}$ or $\mathbb{R}$ and spaces considered which are not manifolds are assumed to be of the homotopy type of $\mathrm{CW}$-complexes such that the function space exponential law holds.

\section{THE $\lambda_{\hat{\alpha}}$-INVARIANT}

An $S^{1}$-action and its orbit map may be generalized to produce certain basic elements in the fundamental groups of $M$ and the function space $\left(M^{M}, 1_{M}\right)$ in the following manner: Say that an element $\alpha \in \pi_{1}(M)$ is in the Gottlieb group of $M$, denoted $\alpha \in G(M)$, if there is an associated map

$$
A: S^{1} \times M \rightarrow M
$$

such that $\left.A\right|_{M}=1_{M}$ and $\left.A\right|_{S^{1}}=\alpha$. We recall some facts about $G(M)\left[\mathrm{Go}_{1}\right]$ :

1. $G(M) \subseteq Z \pi_{1}(M)$.

2. $G(M)$ is a subgroup of the group consisting of all $\alpha \in \pi_{1}(M)$ which act trivially on all $\pi_{*}(M)$ via the usual action of the fundamental group on higher homotopy. Note that this statement incorporates 1 . since $\pi_{1}(M)$ acts on itself via conjugation.

3. If $M=K(\pi, 1)$, then $G(M)=Z \pi_{1}(M)$.

4. Gottlieb's Theorem: If $M$ is a compact $K(\pi, 1)$ and $\chi(M) \neq 0$, then $Z \pi=0$.

By the exponential law, there is a map $\hat{\alpha}: S^{1} \rightarrow M^{M}, \hat{\alpha}(s)(x)=A(s, x)$ such that evaluation $\operatorname{ev}(f)=f(m)$ of a function at a basepoint $m \in M$ entails ev $\circ \hat{\alpha}=\alpha$. Hence, $\mathrm{ev}_{\#}(\hat{\alpha})=\alpha$ where $\mathrm{ev}_{\#}: \pi_{1}\left(M^{M}, 1_{M}\right) \rightarrow \pi_{1}(M)$. In the following we shall keep the notation $A, \alpha, \hat{\alpha}$, etc. Note that a group action $A: S^{1} \times M \rightarrow M$ provides a Gottlieb element $\left.\alpha \simeq A\right|_{S^{1}}$ which may be identified with the homotopy class of the orbit map $a: S^{1} \rightarrow M$. Also note that it is possible for $\alpha$ to be nullhomotopic, but $\hat{\alpha}$ to be essential. When there is no possibility of confusion, we shall use either $\alpha$ or $a$ to denote the orbit map or its homotopy class.

Following $\left[\mathrm{Go}_{2}\right]$, the associated map $A$ or, equivalently, the element $\hat{\alpha} \in$ $\pi_{1}\left(M^{M}, 1_{M}\right)$ may be considered as a clutching map [Sp, p. 455] along the equator of $S^{2}$ which constructs a fibration

$$
M \stackrel{i}{\rightarrow} E \rightarrow S^{2}
$$

with $\alpha=\partial_{\#}(1) \in \operatorname{Im}\left(\partial_{\#}: \pi_{2} S^{2} \rightarrow \pi_{1} M\right)$. Such a fibration has a Wang sequence associated to it,

$$
\cdots \rightarrow H^{q}(E) \stackrel{i^{*}}{\rightarrow} H^{q}(M) \stackrel{\lambda_{\dot{\alpha}}}{\rightarrow} H^{q-1}(M) \rightarrow H^{q+1}(E) \rightarrow \cdots .
$$


The map $\lambda_{\hat{\alpha}}$ is a derivation on $H^{*}(M)$, i.e., satisfies $\lambda_{\hat{\alpha}}(u v)=\lambda_{\hat{\alpha}}(u) v+$ $(-1)^{|u|} u \lambda_{\hat{\alpha}}(v)$, and is called the Wang derivation. Another way to think of this is as follows. There is a classifying space $\operatorname{Baut}(M)$ for fibrations with fibre $M$ which obeys $\pi_{i+1} \operatorname{Baut}(M)=\pi_{i}\left(M^{M}, 1_{M}\right)$. Therefore, $\hat{\alpha} \in \pi_{1}\left(M^{M}, 1_{M}\right)$ corresponds to an element in $\pi_{2} \operatorname{Baut}(M)$ represented by a map $S^{2} \rightarrow \operatorname{Baut}(M)$. The fibration $M \rightarrow E \rightarrow S^{2}$ above is simply the pullback of the classifying fibration over $\operatorname{Baut}(M)$.

There is a beautiful connection between the Wang sequence and the clutching map $A$. Namely, for any $u \in H^{q}(M)$,

$$
A^{*}(u)=1 \times u+\bar{\sigma} \times \lambda_{\hat{\alpha}}(u),
$$

where $\bar{\sigma} \in H^{1}\left(S^{1}\right)$ is a generator, $\alpha=\mathrm{ev}_{\#}(\hat{\alpha})$ and $\times$ is the external product. In case $u \in H^{1}(M)$, we have $\alpha^{*}(u)=\lambda_{\hat{\alpha}}(u) \bar{\sigma}$, with $\lambda_{\hat{\alpha}}(u) \in H^{0}(M)=\mathbb{Q}$, and the expression $(\dagger)$ may be rewritten

$$
\begin{aligned}
A^{*}(u) & =1 \times u+\bar{\sigma} \times \lambda_{\hat{\alpha}}(u) \\
& =1 \times u+\alpha^{*}(u) \times 1 .
\end{aligned}
$$

In $\S 5$ we shall use a more general version of $(\dagger)$. Also, (cf. [Go $2,(10.3)]$ ) for $u \in H^{q}(M ; \mathbb{Z}), \lambda_{\hat{\alpha}}(u)=h(\hat{\alpha}) \backslash \hat{E}^{*}(u)$, where $\backslash$ denotes slant product, $h$ : $\pi_{1}\left(M^{M}, 1_{M}\right) \cong H_{1}\left(M^{M} ; \mathbb{Z}\right)$ is the Hurewicz map and $\hat{E}: M^{M} \times M \rightarrow M$ is the evaluation map $\hat{E}(f, x)=f(x)$. This means that if we consider rational coefficients in cohomology, we have

Lemma 2.1. If $\hat{\alpha} \in \pi_{1}\left(M^{M}, 1_{M}\right)$ has finite order, then $\lambda_{\hat{\alpha}}$ is the zero homomorphism.

Such a slant product description of the Calabi invariant was, in fact, known to symplectic geometers (cf. $\left.\left[\mathrm{McD}_{3}\right]\right)$. We discuss this relationship in $\S 3$. In the sequel we shall be interested in $\lambda_{\hat{\alpha}}(\omega)$ for a c-symplectic manifold $(M, \omega)$. However, for a general space $M$, and any class $\omega \in H^{2}(M)$, we have a basic result, which follows immediately from the exactness of the Wang sequence:

Proposition 2.2. $\lambda_{\hat{\alpha}}(\omega)=0$ if and only if there exists $\bar{\omega} \in H^{2}(E)$ with $i^{*} \bar{\omega}=\omega$.

In order to connect the $\lambda_{\hat{\alpha}}$-invariant to symplectic geometry we require the following fundamental result:

Theorem 2.3. If $\hat{\alpha}$ comes from a symplectic $S^{1}$-action on a symplectic manifold $(M, \omega)$, then $\lambda_{\hat{\alpha}}(\omega)=[i(\underline{X}) \omega]$.

This will follow immediately from the following lemma. Let $p_{1}: S^{1} \times M \rightarrow$ $S^{1}$ and $p_{2}: S^{1} \times M \rightarrow M$ denote, respectively, projection onto the first and second factor and $i_{1}^{m}: S^{1} \rightarrow S^{1} \times M$ and $i_{2}^{t}: S^{1} \rightarrow S^{1} \times M$ be inclusions defined by $i_{1}^{m}(t)=(t, m), i_{2}^{t}(m)=(t, m)$. Note that $p_{j} i_{j}=\mathrm{id}, p_{j} i_{k}=$ constant for $j \neq k, A i_{1}^{m}=A_{m}$ and $A i_{2}^{t}=A_{t}$ where $A_{m}$ is the orbit map at $m, A_{m}(t)=$ $A(t, m)$ and $A_{t}$ is the diffeomorphism at $t, A_{t}(m)=A(t, m)$. Because the $S^{1}$-action is symplectic, $A_{t}^{*} \omega=\omega$ and $A_{t}^{*}(i(\underline{X}) \omega)=i(\underline{X}) \omega$ ([A-M, p. 202]). Finally, for a fixed unit vector $X \in T_{e} S^{1}$ (i.e., a left invariant vector field), let $\nu$ denote the dual left invariant volume form on $S^{1}$ (i.e., $\nu(X)=1$ ) and write $d t$ for its local representation. 
Lemma 2.4. For the symplectic $S^{1}$-action $A$,

$$
A^{*} \omega=p_{2}^{*} \omega+p_{1}^{*} \nu \wedge p_{2}^{*} i(\underline{X}) \omega
$$

where $\underline{X}$ is the fundamental vector field associated to the action.

Proof. Because $T\left(S^{1} \times M\right) \cong T S^{1} \times T M$, it is sufficient to evaluate $A^{*} \omega$ on vector fields of the form $(X, Y)$, where $X$ is as above and $Y$ is any vector field on $M$. Note that $(X, Y)_{(t, m)}=\left(i_{1 *}^{m} X_{t}, i_{2 *}^{t} Y_{m}\right)$. Then (using $A_{m *} X=\underline{X}$ ) we obtain

$$
\begin{aligned}
A^{*} \omega(X, Y)_{(t, m)} & =A^{*} \omega\left(i_{1 *}^{m} X_{t}, i_{2 *}^{t} Y_{m}\right) \\
& =\omega\left(A_{m *} X_{t}, A_{t *} Y_{m}\right) \\
& =i\left(\underline{X}_{t \cdot m}\right) \omega_{t \cdot m}\left(A_{t *} Y_{m}\right) \\
& =A_{t}^{*}\left(i\left(\underline{X}_{t \cdot m}\right) \omega_{t \cdot m}\right)\left(Y_{m}\right) \\
& =i\left(\underline{X}_{m}\right) \omega_{m}\left(Y_{m}\right) .
\end{aligned}
$$

Hence, $A^{*} \omega$ is independent of $t$. This means that, in local coordinates $t, x_{1}$, $\ldots, x_{2 n}$, we may write the 2 -form $A^{*} \omega$ as $A^{*} \omega=p_{2}^{*} \sigma+p_{1}^{*} d t \wedge p_{2}^{*} \beta$. In order to identify $\sigma$, we apply $i_{2}^{i *}$,

$$
\omega=A_{t}^{*} \omega=i_{2}^{t *} A^{*} \omega=i_{2}^{t *} p_{2}^{*} \sigma=\sigma
$$

since $p_{1} i_{2}^{t}$ is constant. Now, in order to identify $\beta$, we apply this local expression for $A^{*} \omega$ to the vector field $(X, Y)$.

$$
\begin{aligned}
A^{*} \omega(X, Y)_{(t, m)}= & p_{1}^{*} d t\left(i_{1 *}^{m} X_{t}\right) \cdot p_{2}^{*} \beta\left(i_{2 *}^{t} Y_{m}\right)-p_{1}^{*} d t\left(i_{2 *}^{t} Y_{m}\right) \cdot p_{2}^{*} \beta\left(i_{1 *}^{m} X_{t}\right) \\
& +p_{2}^{*} \omega\left(i_{1 *}^{m} X_{t}, i_{2 *}^{t} Y_{m}\right) \\
= & d t\left(p_{1 *} i_{1 *}^{m} X_{t}\right) \cdot \beta\left(p_{2 *} i_{2 *}^{t} Y_{m}\right) \\
= & d t\left(X_{t}\right) \cdot \beta\left(Y_{m}\right) \\
= & \beta\left(Y_{m}\right) .
\end{aligned}
$$

Hence, equating the two expressions for $A^{*} \omega(X, Y)_{(t, m)}$, we have $\beta=i(\underline{X}) \omega$. Replacing the local $d t$ by the global volume form $\nu$, we obtain

$$
A^{*} \omega=p_{2}^{*} \omega+p_{1}^{*} \nu \wedge p_{2}^{*} i(\underline{X}) \omega .
$$

Remark 2.5. Another proof of Lemma 2.4 may be given by adapting the proof of the Momentum Lemma of [A-M, p. 288]. Also, the formula of Lemma 2.4 may be generalized for a symplectic toral action (cf. $\S 5$ ) to

$$
A^{*} \omega=p_{2}^{*} \omega+p_{1}^{*} \nu_{1} \wedge p_{2}^{*} i\left(\underline{X}_{1}\right) \omega+\cdots+p_{1}^{*} \nu_{k} \wedge p_{2}^{*} i\left(\underline{X}_{k}\right) \omega+a^{*}(\omega),
$$

where $\underline{X}_{i}$ is the fundamental vector field associated to the $i$ th component circle $S^{1}$ in $T^{k}$ and $\nu_{i}$ is the volume form of that circle. Here, also, $p_{1}$ denotes projection onto $T^{k}$ and $a^{*}$ is the orbit map pullback denoted above by $A_{m}^{*}$. Of course, each of the terms before the last is detected by evaluation on vector fields $\left(X_{i}, Y\right)$ just as above. In the toral case, however, $T^{k}$ has its own 2-forms, so the local expression for $A^{*} \omega$ includes a term which is a linear combination of $d t_{r} \wedge d t_{s}$ for $r, s=1, \ldots, k$. This term may be identified by

$$
\begin{aligned}
A^{*} \omega\left(X_{r}, X_{s}\right)_{(t, m)} & =A^{*} \omega\left(i_{1 *}^{m}\left(X_{r}\right)_{t}, i_{1 *}^{m}\left(X_{s}\right)_{t}\right) \\
& =\omega\left(A_{m *}\left(X_{r}\right)_{t}, A_{m *}\left(X_{s}\right)_{t}\right) \\
& =A_{m}^{*} \omega\left(X_{r}, X_{s}\right) \\
& =a_{m}^{*} \omega\left(X_{r}, X_{s}\right) .
\end{aligned}
$$


Theorem 2.3 now follows from $(\dagger)$ and by the identification of wedge and cup product (which we have written as external product $\times$ ).

Our general approach is now clear. We replace the geometric quantity $i(\underline{X}) \omega$ by the cohomological $\lambda_{\hat{\alpha}}(\omega)$ which, in fact, only requires c-symplecticness for its definition and applicability. With this in mind, we introduce the following terminology:

Definition 2.6. For a c-symplectic manifold $(M, \omega)$, an element $\hat{\alpha} \in$ $\pi_{1}\left(M^{M}, 1_{M}\right)$ is said to be cohomologically Hamiltonian, or c-Hamiltonian, if $\lambda_{\hat{\alpha}}(\omega)=0$. If $\hat{\alpha}$ comes from an $S^{1}$-action and $\lambda_{\hat{\alpha}}(\omega)=0$, then we say that the action is c-Hamiltonian.

Before we go on to the exact results, we recall the notion of fibre number and its ramifications $\left[\mathrm{Go}_{3}\right]$. Suppose $M \stackrel{i}{\rightarrow} E \stackrel{p}{\rightarrow} B$ is a fibration and $M$ is a space with $H^{r}(M ; \mathbb{Z})=0$, for $r>N$ and $H^{N}(M ; \mathbb{Z})=\mathbb{Z}$. Then $i^{*}: H^{N}(E ; \mathbb{Z}) \rightarrow$ $H^{N}(M ; \mathbb{Z})$ has image $k \mathbb{Z}$ for some $k$. The number $k$ is called the fibre number of the fibration $p$ and is denoted by $\Phi(p)$. Two fundamental facts about the fibre number are included in:

Fibre Number Theorem $2.7\left[\mathrm{Go}_{3}\right]$.

1. If $\partial: \Omega B \rightarrow M$ is the connecting map of the fibration, then $0=\Phi(p) \cdot \partial_{*}$ : $H_{*}(\Omega B) \rightarrow H_{*}(M)$. If we only consider rational or real coefficients, then a nonzero fibre number implies $\partial_{*}=0$.

2. If the fibration is the Borel fibration associated to a toral action on an orientable manifold, $M \rightarrow M T^{k} \rightarrow B T^{k}$, then the the fibre number, now denoted $\Phi\left(T^{k}, M\right)$, is nonzero if and only if there exist fixed points for the action.

Note that, while we normally use rational coefficients, these suffice in the consideration of the vanishing or nonvanishing of the fibre number. Also, the first statement above is proved in [Go 3 ] only for the trace of an action, so we shall give a proof below along the lines of the Transgression Theorem of [C-G]. The second statement relies on the definition, properties and analogous result for trace as well as the identification of the trace with the fibre number under the stated conditions. In order to make this paper somewhat self-contained, we also discuss these notions below.

Proof of (1). There is a homotopy commutative diagram

$$
\begin{array}{ccc}
\Omega B \times M & \stackrel{d}{\rightarrow} & M \\
j \times 1_{M} \downarrow & & \downarrow i \\
P B \times M & \stackrel{\hat{d}}{\rightarrow} & E \\
\pi \downarrow & & \downarrow p \\
B & \stackrel{1_{B}}{\rightarrow} & B
\end{array}
$$

where $P B$ denotes the path space of $B, d$ denotes the 'action' of loops on the fibre and $\hat{d}$ is the extension of the action to $P B$. Note that $\left.d\right|_{\Omega B}=\partial$ and $\left.d\right|_{M}=1_{M}$ by general properties of the action. Suppose $\Phi(p) \neq 0$ and let 
$i^{*}(\alpha)=\mu$ where $\mu \in H^{N}(M)$ is the top class of $M$. Now suppose $x \in H^{q}(M)$ is an arbitrary element of positive degree. Then $x \cup \mu=0$ since $\mu$ is a top class and we get

$$
\begin{aligned}
0 & =d^{*}(x \cup \mu) \\
& =d^{*}(x) \cup d^{*}\left(i^{*}(\alpha)\right) \\
& =\left(\partial^{*}(x) \times 1+\sum a_{i} \times b_{i}+1 \times x\right) \cup(j \times 1)^{*} \hat{d}^{*}(\alpha)
\end{aligned}
$$

where the $b_{i}$ have positive degree in $H^{*}(M)$. Now, from the diagram and the contractibility of $P B$ we see that $\hat{d}^{*}(\alpha)=i^{*}(\alpha)$ and $(j \times 1)^{*} \hat{d}^{*}(\alpha)=1 \times i^{*}(\alpha)$. Hence, since $b_{i} \cup i^{*}(\alpha)=b_{i} \cup \mu=0$, we obtain

$$
\begin{aligned}
0 & =\left(\partial^{*}(x) \times 1+\sum a_{i} \times b_{i}+1 \times x\right) \cup(j \times 1)^{*} \hat{d}^{*}(\alpha) \\
& =\partial^{*}(x) \times i^{*}(\alpha) \\
& =\partial^{*}(x) \times \mu .
\end{aligned}
$$

This is true if and only if $\partial^{*}(x)=0$.

The following discussion is culled from [ $\left[\mathrm{Go}_{3}\right]$. Suppose $M \rightarrow E \stackrel{p}{\rightarrow} B$ is a fibration. A transfer for $p$ is a homomorphism $\tau: H_{*}(B ; \mathbb{Z}) \rightarrow H_{*}(E ; \mathbb{Z})$ such that $p_{*} \circ \tau=N \in \mathbb{Z}$, where $N$ denotes the endomorphism of $H_{*}(B ; \mathbb{Z})$ given by multiplication by $N$. It is easy to see that the set of all such $N$ is an ideal in $\mathbb{Z}$ with generator denoted $\operatorname{deg}(p)$ and called the degree of $p$. The relation between $\operatorname{deg}(p)$ and the fibre number $\Phi(p)$ is via integration(s) over the fibre $p_{\#}: H_{i}(B ; \mathbb{Z}) \rightarrow H_{m+i}(E ; \mathbb{Z})$ and $p^{\#}: H^{i}(E ; \mathbb{Z}) \rightarrow H^{i-m}(B ; \mathbb{Z})$. (In the presence of Poincare duality, the Umkehrhomomorphisms $p_{1}$ and $p^{\text {! }}$ may be used.) Namely, by the definition of fibre number, there exists $x \in$ $H^{m}(E ; \mathbb{Z})$ with $i^{*}(x)=\Phi(p)[F]$ where $[F]$ is the top class of $F$. Define a homomorphism $\tau: H_{i}(B ; \mathbb{Z}) \rightarrow H_{i}(E ; \mathbb{Z})$ by $\tau(y)=x \cap p_{\#}(y)$ and use the fundamental Umkehrungs formula to compute

$$
p_{*} \tau(y)=p_{*}\left(x \cap p_{\#}(y)\right)=p^{\#}(x) \cap y=\Phi(p) \cdot 1 \cap y=\Phi(p) y .
$$

Thus, $\tau$ is a transfer and, by definition of degree, $\operatorname{deg}(p) \mid \Phi(p)$. In fact, if the fibration is a fibre bundle of closed connected orientable manifolds, then Poincare duality and the formula $i_{*}[\bar{F}]=p^{*}[B] \cap[\bar{E}]$ combine to show that $\operatorname{deg}(p)=\Phi(p)$. The trace of $p, \operatorname{tr}(p)$, is defined as the least common multiple of the degrees of all pullbacks of $p$. Since, for any pullback $p^{\prime}$ of $p$, we have $\operatorname{deg}\left(p^{\prime}\right)\left|\Phi\left(p^{\prime}\right)\right| \Phi(p)$, the least common multiple of the degrees (i.e., $\left.\operatorname{tr}(p)\right)$ also divides $\Phi(p)$. Since, for a manifold bundle, $\Phi(p)=\operatorname{deg}(p)$ and $\operatorname{deg}(p) \mid \operatorname{tr}(p)$ by definition, in this case $\operatorname{tr}(p)=\Phi(p)$.

The trace of an action of $G$ on $M, \operatorname{tr}(G, M)$, is defined to be the trace of the associated Borel fibration $M \rightarrow M G \rightarrow B G$. Gottlieb shows that the Borel fibration may be 'approximated' up to a requisite dimension by a manifold fibre bundle having the same trace and fibre number as the Borel fibration. By what we have said above, we see that $\operatorname{tr}(G, M)=\Phi(G, M)$. The advantage of using trace is that there are divisibility properties which characterize certain actions. In particular, if $H$ is a subgroup of $G$, then $\operatorname{tr}(H, M) \mid \operatorname{tr}(G, M)$. Also, if $G$ is a finite group, then $\operatorname{tr}(G, M)=|G|$ if and only if the action is free. Now let's see how these properties of trace allow us to prove the more important direction of the Fibre Number Theorem. Suppose $\Phi(T, M)=\operatorname{tr}(T, M) \neq 0$ where $T$ 
is a torus. Choose a generator $t$ for the torus $T$ and find a sequence of points $t_{p}$ converging to $t$ such that $t_{p}$ has order $p$ a prime. Each $t_{p}$ generates a $\mathbb{Z} / p$ action with $\mathbb{Z} / p \subseteq T$. Therefore, $\operatorname{tr}(\mathbb{Z} / p, M) \mid \operatorname{tr}(T, M) \neq 0$. But $\operatorname{tr}(T, M)$ is finite, so only finitely many $p$ divide it properly. Hence, $\operatorname{tr}(\mathbb{Z} / p, M)=1$ for all $p$ large enough (e.g., $p>\operatorname{tr}(T, M)$ ). By the characterization of free actions above, each of these $t_{p}$ have fixed points. These fixed points, in turn, have limit points as $p \rightarrow \infty$ which must then be fixed by $t$. Hence, these limit points are fixed points of the action of $T$ on $M$.

Before we end this section, we point out that the implication $\Phi(T, M) \neq$ $0 \Rightarrow$ Fixed Point has a direct and simple proof in the case of a circle action. Namely, suppose there are no fixed points for an $S^{1}$-action on $M$. Then $S^{1}$ acts with finite isotropy, so (using rational coefficients) $H^{*}\left(M S^{1}\right) \cong H^{*}\left(M / S^{1}\right)$ and $M / S^{1}$ has dimension $\operatorname{dim}(M)-1$. Thus the cohomological image in degree $\operatorname{dim}(M)$ is zero and the fibre number vanishes. This argument furnishes a direct proof of Corollary 3.7 below. It does not work directly for toral actions because non-existence of fixed points does not automatically imply finite isotropy, but an argument of V. Ginzburg often reduces this situation to that of a circle action (cf. Theorem 5.2).

\section{Properties of the $\lambda_{\hat{\alpha}}$-INVARIANT}

Throughout this section let $M$ be an $N$-dimensional manifold, with top class $\mu \in H^{N}(M ; \mathbb{Q})$. For applications we will specialize to a c-symplectic manifold $\left(M^{2 n}, \omega\right)$, when we can take $\mu=\omega^{n}$. In general, we take $\hat{\alpha} \in$ $\pi_{1}\left(M^{M}, 1_{M}\right)$ and $\alpha=\mathrm{ev}_{\#}(\hat{\alpha}) \in \pi_{1}(M)$. Recall that the Hurewicz homomorphism $h: \pi_{1} M \rightarrow H_{1}(M)$ is defined by $h(\gamma)=\gamma_{*}(\sigma)$, where $\sigma$ is a generator of $H_{1}\left(S^{1} ; \mathbb{Z}\right)$ dual to $\bar{\sigma} \in H^{1}\left(S^{1} ; \mathbb{Z}\right)$ and $\gamma_{*}$ is the map on homology induced by $\gamma: S^{1} \rightarrow M$.

Our initial goal is to clarify the relationship between $\lambda_{\hat{\alpha}}$ and $h(\alpha)$. First, recall that Poincare duality may be characterized in terms of cup, cap and Kronecker products: For $a \in H_{q}(M), z \in H_{N}(M)$ a fundamental class Kronecker dual to the top class $\mu$, the element $\hat{a} \in H^{N-q}(M)$ is Poincaré dual to $a$ if

$$
\langle b \cup \hat{a}, z\rangle=\langle b, \hat{a} \cap z\rangle=\langle b, a\rangle,
$$

for any $b \in H^{q}(M)$. If $u \in H^{1}(M)$, then $u \cup \mu=0$ by dimensional considerations. Since $\lambda_{\hat{\alpha}}$ is a derivation, we get

$$
0=\lambda_{\hat{\alpha}}(u \cup \mu)=\lambda_{\hat{\alpha}}(u) \cdot \mu-u \cup \lambda_{\hat{\alpha}}(\mu) .
$$

Since $u$ is in degree 1 , we have $\alpha^{*}(u)=\lambda_{\hat{\alpha}}(u) \cdot \bar{\sigma}$. Hence,

$$
\begin{aligned}
\left\langle u \cup \lambda_{\hat{\alpha}}(\mu), z\right\rangle & =\left\langle\lambda_{\hat{\alpha}}(u) \cdot \mu, z\right\rangle=\lambda_{\hat{\alpha}}(u) \\
& =\left\langle\alpha^{*}(u), \sigma\right\rangle=\left\langle u, \alpha_{*}(\sigma)\right\rangle=\langle u, h(\alpha)\rangle .
\end{aligned}
$$

Our first result follows immediately from these observations.

Lemma 3.1. Let $\mu \in H^{N}(M)$ be a top class and let $\hat{\alpha} \in \pi_{1}\left(M^{M}, 1_{M}\right)$ with $\alpha=\mathrm{ev}_{\#}(\hat{\alpha})$.

1. For $u \in H^{1}(M), \lambda_{\hat{\alpha}}(u)=\langle u, h(\alpha)\rangle$.

2. $\lambda_{\hat{\alpha}}(\mu)$ is Poincaré dual to $h(\alpha)$.

Combining Lemma 3.1 with another general observation obtains the following result: 
Proposition 3.2. Let $\mu \in H^{N}(M)$ be a top class and let $\hat{\alpha} \in \pi_{1}\left(M^{M}, 1_{M}\right)$ with $\alpha=\mathrm{ev}_{\#}(\hat{\alpha})$. We have equivalences as follows:

$$
\lambda_{\hat{\alpha}}(\mu)=0 \Leftrightarrow \lambda_{\hat{\alpha}}\left(H^{1}(M)\right)=0 \Leftrightarrow h(\alpha)=0 .
$$

Proof. The first equivalence follows from a general result: If $\theta$ is a degree -1 derivation on a Poincare duality algebra $H$, of top dimension $N$, then $\theta\left(H^{N}\right)=0$ if, and only if, $\theta\left(H^{1}\right)=0$ (cf. [L-O, Lemma 3.3]). The second equivalence follows from Lemma 3.1(1).

This gives our first result concerning c-symplecticness:

Proposition 3.3. Let $\left(M^{2 n}, \omega\right)$ be a c-symplectic manifold, with top class $\omega^{n}$, and let $\hat{\alpha} \in \pi_{1}\left(M^{M}, 1_{M}\right)$ with $\alpha=\mathrm{ev}_{\#}(\hat{\alpha})$. If $\hat{\alpha}$ is c-Hamiltonian, i.e., if $\lambda_{\hat{\alpha}}(\omega)=0$, then the three equivalent conditions of Proposition 3.2 hold.

Proof. Since $\lambda_{\hat{\alpha}}$ is a derivation, we have $\lambda_{\hat{\alpha}}\left(\omega^{n}\right)=n \omega^{n-1} \lambda_{\hat{\alpha}}(\omega)=0$.

Recall that a c-symplectic manifold $\left(M^{2 n}, \omega\right)$ has Lefschetz type if the multiplication homomorphism $\omega^{n-1}: H^{1}(M) \rightarrow H^{2 n-1}(M)$ is an isomorphism. The Lefschetz type hypothesis allows for the implication of Proposition 3.3 to be reversed:

Theorem 3.4. Let $\left(M^{2 n}, \omega\right)$ be a c-symplectic manifold of Lefschetz type and let $\hat{\alpha} \in \pi_{1}\left(M^{M}, 1_{M}\right)$ with $\alpha=\mathrm{ev}_{\#}(\hat{\alpha})$. Then the following are equivalent:

1. The element $\hat{\alpha}$ is $c$-Hamiltonian, i.e., $\lambda_{\hat{\alpha}}(\omega)=0$.

2. $\lambda_{\hat{\alpha}}\left(H^{1}(M)\right)=0$.

3. $h(\alpha)=0$.

Proof. In light of Proposition 3.3, we need only show that $h(\alpha)=0$ implies $\lambda_{\hat{\alpha}}(\omega)=0$. Now, by Lemma 3.1, $h(\alpha)$ is Poincaré dual to $\lambda_{\hat{\alpha}}\left(\omega^{n}\right)=n \cdot \lambda_{\hat{\alpha}}(\omega) \cup$ $\omega^{n-1}$. By duality, $h(\alpha)=0$ then implies $n \cdot \lambda_{\hat{\alpha}}(\omega) \cup \omega^{n-1}=0$ and, by the Lefschetz type hypothesis, this can only happen if $\lambda_{\hat{\alpha}}(\omega)=0$.

Revert to the general case of a manifold $M$, and consider an $S^{1}$-action on $M$ with $\hat{\alpha} \in \pi_{1}\left(M^{M}, 1_{M}\right)$ represented by the adjoint map of the action $\hat{\alpha}: S^{1} \rightarrow M^{M}$. The action is homotopically encoded in the Borel fibration (and its Barratt-Puppe extension)

$$
S^{1} \stackrel{a}{\rightarrow} M \stackrel{j}{\rightarrow} M S^{1} \rightarrow B S^{1} .
$$

Now, because $A: S^{1} \times M \rightarrow M$ is an action, the adjoint map $\hat{\alpha}: S^{1} \rightarrow M^{M}$ is an $H$-map and so induces a map of classifying spaces $B S^{1} \rightarrow \operatorname{Baut}(M)$ which at the $\pi_{2}$-level is simply the map $S^{2} \rightarrow \operatorname{Baut}(M)$ classifying the Wang fibration associated to $\hat{\alpha}$. The factorization $S^{2} \rightarrow B S^{1} \rightarrow \operatorname{Baut}(M)$ then provides a homotopy-commutative diagram

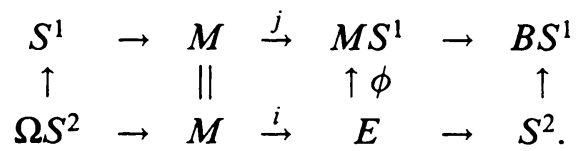

Each fibration has an associated long exact sequence in homotopy and the diagram induces a commutative ladder

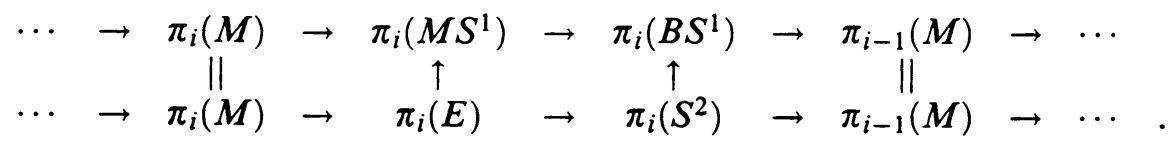


A simple diagram chase shows that, for $i=0,1$ or $2, \pi_{i} E \cong \pi_{i} M S^{1}$ and also $\pi_{3} E \rightarrow \pi_{3} M S^{1}$ is surjective. Applying the Hurewicz theorem and duality, we obtain the following result:

Lemma 3.5. Let $A: S^{1} \times M \rightarrow M$ be an action. With the above notation, $\phi^{*}: H^{r}\left(M S^{1}\right) \rightarrow H^{r}(E)$ is an isomorphism for $r \leq 2$.

For actions on a c-symplectic manifold, this has the following implication (cf. $[\mathrm{A}-\mathrm{B}]$ ):

Theorem 3.6. Let $A: S^{1} \times M \rightarrow M$ be an action on a c-symplectic manifold $\left(M^{2 n}, \omega\right)$. The action is $c$-Hamiltonian, i.e., $\lambda_{\hat{\alpha}}(\omega)=0$, if and only if there exists $\tilde{\omega} \in H^{2}\left(M S^{1}\right)$ with $j^{*} \tilde{\omega}=\omega$.

Proof. We have seen in Proposition 2.2 that $\lambda_{\hat{\alpha}}(\omega)=0$ if and only if there exists $\bar{\omega} \in H^{2}(E)$ with $i^{*} \bar{\omega}=\omega$. The isomorphism on $H^{2}$ obtained from Lemma 3.5 and the homotopy-commutativity of the diagram, i.e., $\phi i \simeq j$, now imply the result.

Corollary 3.7. A c-Hamiltonian action on a c-symplectic manifold has fixed points.

Proof. Since the action is c-Hamiltonian, then $\lambda_{\hat{\alpha}}(\omega)=0$. By Theorem 3.6, there exists $\tilde{\omega} \in H^{2}\left(M S^{1}\right)$ with $j^{*} \tilde{\omega}=\omega$. Then we have $j^{*} \tilde{\omega}^{n}=\omega^{n} \neq 0$ and the fibre number $\Phi\left(S^{1}, M\right)$ is nonzero. By the Fibre Number Theorem, the action has fixed points.

Of course a fixed point for an action entails the triviality of the homology homomorphism $\alpha_{*}: H_{1}\left(S^{1}\right) \rightarrow H_{1}(M)$ induced by the orbit map $\alpha: S^{1} \rightarrow M$. Indeed, if the action has a fixed point, then $\alpha=0 \in \pi_{1} M$. This in turn gives $h(\alpha)=\alpha_{*}(\sigma)=0$. By our previous results on Lefschetz type, we obtain the following theorem:

Theorem 3.8. Let $\left(M^{2 n}, \omega\right)$ be a c-symplectic manifold of Lefschetz type. Let $A: S^{1} \times M \rightarrow M$ be an $S^{1}$-action on $M$ with adjoint $\hat{\alpha} \in \pi_{1}\left(M^{M}, 1_{M}\right)$ and orbit map $\alpha$. Then the following are equivalent:

1. The action is c-Hamiltonian, i.e., $\lambda_{\hat{\alpha}}(\omega)=0$.

2. $\lambda_{\hat{\alpha}}\left(H^{1}(M)\right)=0$.

3. The action has fixed points.

4. $h(\alpha)=0$.

Of course Ono's Theorem (Theorem A of the Introduction) follows for a symplectic circle action by the identification $\lambda_{\hat{\alpha}}(\omega)=[i(\underline{X}) \omega]$. Also, the equivalence of 2. and 3. is a version of [C- $R_{1}$, Lemma 12.7]. The connection is made apparent by realizing that the Wang derivation $\lambda_{\hat{\alpha}}$ is essentially the $d^{2}$ of the Serre spectral sequence.

Finally, there is an interpretation, due to Gottlieb [Go ${ }_{2}$, Theorem 13.1], of $\lambda_{\hat{\alpha}}(\omega)$ as an obstruction. Let $K(\mathbb{Q}, 1) \rightarrow P \stackrel{p}{\rightarrow} M$ denote the principal fibration corresponding to $\omega \in H^{2}(M ; \mathbb{Q})$ and let $A: S^{1} \times M \rightarrow M$ be the adjoint of $\hat{\alpha} \in \pi_{1}\left(M^{M}, 1_{M}\right)$.

Theorem 3.9. $\lambda_{\hat{\alpha}}(\omega)=0$ if and only if there exists a map $\bar{A}: S^{1} \times P \rightarrow P$ such that $\left.\bar{A}\right|_{P}=1_{P}$ and the following diagram commutes: 


$\begin{array}{ccc}S^{1} \times P & \stackrel{\bar{A}}{\rightarrow} & P \\ 1 \times p \downarrow & & \downarrow p \\ S^{1} \times M & \stackrel{A}{\rightarrow} & M .\end{array}$

If $\omega \in H^{2}(M ; \mathbb{Z})$ and $A$ is an $S^{1}$-action, then the vanishing of the integral class $\lambda_{\hat{\alpha}}(\omega)$ is equivalent to the existence of a lifting of the $S^{1}$-action to the $S^{1}$-principal bundle determined by $\omega\left[\mathbf{G o}_{4}\right]$. Being able to lift an action to the principal bundle determined by the form then implies that the action is Hamiltonian. If, on the other hand, we know that say, $H^{1}(M ; \mathbb{Z})$ has no torsion, then Hamiltonian in turn, implies the existence of a lifting.

In [Go ${ }_{2}$, Theorem 8.8] Gottlieb proved that any characteristic class $k$ of the tangent bundle $T M$ always has $\lambda_{\hat{\alpha}}(k)=0$ (even integrally). To apply this to symplectic manifolds, we note that, because $S^{1}$ acts symplectically on $M$, each $g \in S^{1}$ lifts to a bundle map $d g: T M \rightarrow T M$ which on a fibre belongs to $S p(2 n, \mathbb{R})$. This also means that the universal bundle in Gottlieb's result has base $B S p(2 n, \mathbb{R}) \simeq B U(n)($ since $U(n) \subset S p(2 n, \mathbb{R})$ is a deformation retract) and the universal Chern classes pull back via

$$
S^{1} \times M \stackrel{A}{\rightarrow} M \stackrel{\tau}{\rightarrow} B U(n)
$$

where $\tau: M \rightarrow B U(n)$ classifies the tangent bundle. A particular case of Gottlieb's Theorem then says

Theorem 3.10 [ $\mathrm{Go}_{2}$, Corollary 8.6]. If $k \in H^{*}(B U(n) ; \mathbb{Q})$, then

$$
A^{*}\left(\tau^{*}(k)\right)=1 \times \tau^{*}(k) \in H^{*}\left(S^{1} \times M ; \mathbb{Q}\right) .
$$

For $k=c_{1}$, the first universal Chern class, $\tau^{*}(k)=c_{1}(M)$ since $\tau$ classifies the tangent bundle. Theorem 3.10 then gives $\lambda_{\hat{\alpha}}\left(c_{1}(M)\right)=0$ and we are led to

Theorem 3.11. Suppose the first Chern class of a symplectic manifold $(M, \omega)$ is a nonzero multiple of $\omega, c_{1}(M)=r \cdot \omega$. Then any symplectic $S^{1}$-action on $M$ is Hamiltonian.

As mentioned in $\S 1$, Dusa $\mathrm{McDuff}\left[\mathrm{McD}_{3}\right]$ used essentially the same argument to prove the vanishing of the so-called Calabi invariant under the hypothesis $c_{1}(M)=r \cdot \omega$. Because McDuff worked in a geometric context, her task was to relate the geometrically defined Calabi invariant to the homotopical work of Gottlieb. From our point of view, the relation of $\lambda_{\hat{\alpha}}$ to Gottlieb's lifting results is almost tautological, so once the identification of Theorem 2.3, i.e., $[i(\underline{X}) \omega]=\lambda_{\hat{\alpha}}(\omega)$, is made, results such as Theorem 3.11 seem quite natural.

Recall from [Cal] and [Ban] that a path $\hat{\alpha}: I \rightarrow G_{\text {sym }}(M)$ with $\hat{\alpha}(0)=1_{M}$, where $G_{\text {sym }}(M)$ denotes the identity component of the group of symplectic diffeomorphisms of $M$, has associated with it a family of vector fields defined by

$$
X_{t}(p)=\frac{d \hat{\alpha}_{t}}{d t}\left(\hat{\alpha}_{t}^{-1}(p)\right)
$$

where $p \in M$. In Banyaga's notation, a closed 1 -form is defined by $\Sigma\left(\hat{\alpha}_{t}\right)=$ $\int_{0}^{1} i\left(X_{t}\right) \omega d t$ which only depends on the homotopy class, relative the endpoints, 
of $\hat{\alpha}$. Now the usual covering space construction allows points of the universal cover of $G_{\text {sym }}(M)$ to be written as $(h,[\hat{\alpha}])$ where $h \in G_{\text {sym }}(M)$ and $\hat{\alpha}(1)=$ $h$. Denoting the universal cover by $\tilde{G}_{\text {sym }}(M)$ the Calabi invariant may be defined as the homomorphism $S: \tilde{G}_{\mathrm{sym}}(M) \rightarrow H^{1}(M ; \mathbb{R})$ with $S(h,[\hat{\alpha}])=$ $[\Sigma(\hat{\alpha})]$. (This map is usually denoted $\tilde{S}$ while $S$ is reserved for the induced homomorphism of $G_{\text {sym }}(M)$ into a certain quotient of $H^{1}(M ; \mathbb{R})$. We will have no need for this homomorphism.)

Now take a loop $\hat{\alpha}: S^{1} \rightarrow G_{\text {sym }}(M)$ based at $1_{M}$ together with its adjoint map $A: S^{1} \times M \rightarrow M$. We think of $\hat{\alpha}$ as representing a class in $\pi_{1}\left(G_{\text {sym }}(M), 1_{M}\right)$. Let $\Gamma: S^{1} \rightarrow M$ be a 1 -cycle with homology class $\gamma \in$ $H_{1}(M)$. We have a map

$$
S^{1} \times S^{1} \stackrel{1 \times \Gamma}{\rightarrow} S^{1} \times M \stackrel{A}{\rightarrow} M
$$

which gives a 2-cycle $A(\Gamma)$ with $A(\Gamma)(s, t)=\hat{\alpha}(t)(\Gamma(s))$ and a homology class which we denote by $\lambda_{\alpha}^{*}(\gamma) \in H_{2}(M)$. Banyaga shows

$$
\int_{A(\Gamma)} \omega=\int_{S^{1} \times S^{1}} A(\Gamma)^{*} \omega=\int_{\Gamma} \Sigma(\hat{\alpha}) .
$$

This may be thought of in an equivalent, but more algebraic way: let $\sigma \in H_{1}\left(S^{1}\right)$ be a chosen generator and form $\sigma \times \gamma \in H_{2}\left(S^{1} \times M\right)$. Then Gottlieb shows in $\left[\mathrm{Go}_{6}\right]$ that

$$
A_{*}(\sigma \times \gamma)=\lambda_{\alpha}^{*}(\gamma)
$$

is dual to $\lambda_{\hat{\alpha}}(\omega)$ in the Kronecker product sense,

$$
\left\langle\omega, \lambda_{\hat{\alpha}}^{*}(\gamma)\right\rangle=\left\langle\lambda_{\hat{\alpha}}(\omega), \gamma\right\rangle .
$$

Then, thinking of $S(\hat{\alpha})$ as an element of $\operatorname{Hom}\left(H_{1}(M), \mathbb{R}\right)$, we have

$$
\begin{aligned}
S(\hat{\alpha})(\gamma) & =\int_{A(\Gamma)} \omega=\langle\omega,[A(\Gamma)]\rangle \\
& =\left\langle\omega, \lambda_{\hat{\alpha}}^{*}(\gamma)\right\rangle=\left\langle\lambda_{\hat{\alpha}}(\omega), \gamma\right\rangle .
\end{aligned}
$$

Lemma 3.12. $S(\hat{\alpha})=\lambda_{\hat{\alpha}}(\omega)$ for $\hat{\alpha} \in \pi_{1}\left(G_{\text {sym }}(M), 1_{M}\right)$.

So, in the symplectic case, we see that c-Hamiltonian correponds to zero Calabi invariant. Lemma 3.12 is implicit in $\left[\mathrm{McD}_{3}\right]$ via the slant product descriptions of $S(\hat{\alpha})$ and $\lambda_{\hat{\alpha}}(\omega)$. We would now like to present an interesting sufficient condition for a loop $\hat{\alpha}$ to be c-Hamiltonian. This condition was pointed out to us by Viktor Ginzburg and is an analogue of his Assertion $1 \mathrm{~b}$. in [ $\mathrm{Gi}_{2}$ ]. Since his result uses the discreteness of $S\left(\pi_{1}\left(G_{\mathrm{sym}}(M), 1_{M}\right)\right)$ in the Lefschetz type case, we first discuss this by modifying the arguments of [Ban] and $\left[\mathrm{McD}_{3}\right]$ to fit the $\lambda_{\hat{\alpha}}$-invariant.

Let $\Gamma_{\omega}$ denote the image of $S: \pi_{1}\left(G_{\text {sym }}(M), 1_{M}\right) \rightarrow H^{1}(M)$. By the above, we see that

$$
\Gamma_{\omega}=\left\{\lambda_{\hat{\alpha}} \omega \mid \hat{\alpha} \in \pi_{1}\left(G_{\text {sym }}(M), 1_{M}\right)\right\} .
$$

Let $\Gamma_{\omega^{n}}=\left\{\lambda_{\hat{\alpha}} \omega^{n} \mid \hat{\alpha} \in \pi_{1}\left(G_{\text {sym }}(M), 1_{M}\right)\right\}$. Now, $\Gamma_{\omega^{n}} \subseteq H^{2 n-1}(M ; \mathbb{R})$ is discrete since a multiple of $\omega^{n}$ is integral. We also know that $\lambda_{\hat{\alpha}}\left(\omega^{n}\right)=$ $n \lambda_{\hat{\alpha}}(\omega)\left(\omega^{n-1}\right)$, so the inverse of the Lefschetz type isomorphism $u \mapsto n \cdot u \omega^{n-1}$ takes $\lambda_{\hat{\alpha}}\left(\omega^{n}\right)$ to $\lambda_{\hat{\alpha}}(\omega)$. Since this holds for each $\hat{\alpha}, \Gamma_{\omega^{n}}$ is carried isomorphically onto $\Gamma_{\omega}$. Hence, $\Gamma_{\omega}$ is discrete as well. We state this formally as 
Lemma 3.13 [Ban, $\mathrm{McD}_{3}$ ]. If $(M, \omega)$ is a symplectic manifold of Lefschetz type, then

$$
\begin{aligned}
\Gamma_{\omega} & =\left\{\lambda_{\hat{\alpha}} \omega \mid \hat{\alpha} \in \pi_{1}\left(G_{\mathrm{sym}}(M), 1_{M}\right)\right\} \\
& =S\left(\pi_{1}\left(G_{\mathrm{sym}}(M), 1_{M}\right)\right)
\end{aligned}
$$

is discrete in $H^{1}(M)$.

This fact has the following consequence due to V. Ginzburg (compare [ $\left.\mathrm{Gi}_{2}\right]$ ):

Theorem 3.14. Let $\hat{\alpha} \in \pi_{1}\left(G_{\mathrm{sym}}(M), 1_{M}\right)$ with associated family of vector fields $X_{t}$. If $i\left(X_{t}\right) \omega$ is sufficiently $C^{0}$-small for all $t$ and $(M, \omega)$ has Lefschetz type, then $\hat{\alpha}$ is c-Hamiltonian.

Proof. Because $i\left(X_{t}\right) \omega$ is $C^{0}$-small for all $t, \Sigma\left(\hat{\alpha}_{t}\right)$ remains small. Choosing a basis of cycles $\Gamma_{1}, \ldots, \Gamma_{k}$ for $H_{1}(M)$, we evaluate

$$
S(\hat{\alpha})\left(\gamma_{i}\right)=\int_{\Gamma_{i}} \Sigma\left(\hat{\alpha}_{t}\right) .
$$

This can be made arbitrarily small provided $i\left(X_{t}\right) \omega$ is sufficiently $C^{0}$-small for all $t$. Now, the map $\psi: H^{1}(M) \rightarrow \mathbb{R}^{k}$,

$$
\psi([\tau])=\left(\int_{\Gamma_{1}} \tau, \ldots, \int_{\Gamma_{k}} \tau\right)
$$

is a linear isomorphism, so $\psi\left(\Gamma_{\omega}\right)$ is discrete since $\Gamma_{\omega}$ is. Therefore, if $i\left(X_{t}\right) \omega$ is sufficiently $C^{0}$-small for all $t$, then $\psi(S(\hat{\alpha}))=0$. Consequently, $S(\hat{\alpha})=0$ and, by the identification $S(\hat{\alpha})=\lambda_{\hat{\alpha}}(\omega)$, we see that $\lambda_{\hat{\alpha}}(\omega)=0$ as well.

Remark 3.15. The condition on $i\left(X_{t}\right) \omega$ will hold, for example, if $\hat{\alpha}$ is $C^{1}$-close to $1_{M}$ in $\pi_{1}\left(G_{\text {sym }}(M), 1_{M}\right)$ equipped with the $C^{1}$-topology. Also, the same result holds when $\omega$ is an integral class, since in this case it is obvious that $\Gamma_{\omega}$ is discrete. Finally, V. Ginzburg has noted that a loop in $\pi_{1}\left(G_{\mathrm{sym}}(M), 1_{M}\right)$ may be c-Hamiltonian even when none of the individual diffeomorphisms is exact (or $\mathrm{HI}$ in the terminology of [ $\left.\mathrm{Gi}_{2}\right]$ ). Ginzburg's example is the time-dependent rotation of the torus $T^{2}$ given by $(\phi, \theta) \mapsto(\phi, \theta+b(t))$ where $b(t)$ is a bump function.

\section{THE $\left.\omega\right|_{\pi_{2} M}=0$ CASE}

In this section we study the hypothesis of the (section) title from the viewpoint of classical algebraic topology. The first several results apply quite generally to any space. Beginning with Corollary 4.6 , we specialize to the case of a c-symplectic manifold. Any class $\omega \in H^{2}(M ; \mathbb{Q})$ can be thought of as a homomorphism $\omega: H_{2}(M ; \mathbb{Z}) \rightarrow \mathbb{Q}$, by the universal coefficient theorem. Composing the Hurewicz homomorphism with such a class gives a map

$$
\omega \circ h: \pi_{2}(M) \rightarrow \mathbb{Q} \text {. }
$$

We denote the situation when $\omega \circ h=0$ by $\left.\omega\right|_{\pi_{2} M}=0$. The condition $\left.\omega\right|_{\pi_{2} M}=$ 0 makes its appearance in many places in symplectic topology (e.g., $\left[\mathrm{Fl}, \mathrm{On}_{2}\right]$ ) and we hope here to indicate some cohomological reasons for this. Below, for any space $M$, we shall use the following notation: The fundamental group $\pi_{1} M$ shall be denoted $\pi$ and the classifying map of the universal cover shall be 
denoted $f: M \rightarrow K(\pi, 1)$. Also, we shall denote the Hurewicz map in degree $n$ by $h_{n}: \pi_{n} M \rightarrow H_{n}(M ; \mathbb{Z})$. To begin, we recall the simpler portion of Hopf's Theorem:

Theorem 4.1. The classifying map $f: M \rightarrow K(\pi, 1)$ induces isomorphisms with integral coefficients

$$
H_{1}(M ; \mathbb{Z}) \cong H_{1}(K(\pi, 1) ; \mathbb{Z}) \quad \text { and } \quad \frac{H_{2}(M ; \mathbb{Z})}{\left(\operatorname{Im} h_{2}\right)} \cong H_{2}(K(\pi, 1) ; \mathbb{Z}) .
$$

Proof. Clearly $H_{1}(M ; \mathbb{Z}) \cong \pi /[\pi, \pi] \cong H_{1}(K(\pi, 1) ; \mathbb{Z})$, so we just consider the second case. Let $K=K(\pi, 1)$, treat $f$ as an inclusion and consider the Hurewicz ladder

$$
\begin{aligned}
& 0 \quad \rightarrow \quad \pi_{3}(K, M) \stackrel{\cong}{\rightrightarrows} \pi_{2} M \quad \rightarrow \quad 0 \\
& \downarrow \quad \downarrow h_{3} \quad \downarrow h_{2} \quad \downarrow \\
& H_{3}(K ; \mathbb{Z}) \rightarrow H_{3}(K, M ; \mathbb{Z}) \stackrel{\partial_{*}}{\rightarrow} H_{2}(M ; \mathbb{Z}) \stackrel{f_{*}}{\rightarrow} H_{2}(K ; \mathbb{Z}) \rightarrow 0 .
\end{aligned}
$$

Since $\pi_{2} K=0$, then $\pi_{2}(K, M)=0$ also. The relative Hurewicz theorem now says that $H_{1}(K, M ; \mathbb{Z})=0=H_{2}(K, M ; \mathbb{Z})$ and that $h_{3}$ is a surjection. Hence the diagram gives $\operatorname{Im} \partial_{*}=\operatorname{Im}\left(\partial_{*} \circ h_{3}\right)=\operatorname{Im}\left(h_{2} \circ \partial_{\#}\right)=\operatorname{Im} h_{2}$. Therefore,

$$
H_{2}(K ; \mathbb{Z}) \cong \frac{H_{2}(M ; \mathbb{Z})}{\left(\operatorname{Im} \partial_{*}\right)} \cong \frac{H_{2}(M ; \mathbb{Z})}{\left(\operatorname{Im} h_{2}\right)} \text {. }
$$

This allows for a convenient rephrasing of the condition $\left.\omega\right|_{\pi_{2} M}=0$. In the following, note that we have reverted to rational coefficients for cohomology.

Lemma 4.2. $\left.\omega\right|_{\pi_{2} M}=0$ if and only if there exists $\omega_{\pi} \in H^{2}(K)$ with $f^{*} \omega_{\pi}=\omega$. Proof. Suppose that there is an $\omega_{\pi}$ with $f^{*}\left(\omega_{\pi}\right)=\omega$. From the above proof we have that $\operatorname{Im} h_{2}=\operatorname{Im} \partial_{*}$, so that $f_{*} \circ h_{2}=0$. Thus for any $\gamma \in \pi_{2}(M)$ we have $\omega \circ h_{2}(\gamma)=\left(f^{*}\left(\omega_{\pi}\right)\right) \circ h_{2}(\gamma)=\omega_{\pi} \circ f_{*} h_{2}(\gamma)=0$. Hence $\left.\omega\right|_{\pi_{2} M}=0$.

On the other hand, suppose that $\left.\omega\right|_{\pi_{2} M}=0$, i.e., that $\omega \circ h_{2}=0$. Define $\omega_{\pi} \in \operatorname{Hom}\left(H_{2}(K ; \mathbb{Z}), \mathbb{Q}\right)$ as follows: From the diagram in the above proof we have that $f_{*}: H_{2}(M ; \mathbb{Z}) \rightarrow H_{2}(K ; \mathbb{Z})$ is onto. So for $x \in H_{2}(K ; \mathbb{Z})$, set $\omega_{\pi}(x)=\omega(y)$ for any $y \in H_{2}(M ; \mathbb{Z})$ for which $f_{*}(y)=x$. Also from the above proof, with the assumption that $\left.\omega\right|_{\pi_{2} M}=0$, it follows that $\omega_{\pi}$ is well-defined, and we have $f^{*}\left(\omega_{\pi}\right)=\omega$ by construction.

Remark 4.3. The lemma also translates the condition $\left.\omega\right|_{\pi_{2} M}=0$ into a condition on the fundamental group. Since a finite group has trivial rational cohomology, it follows from Lemma 4.2 that a c-symplectic manifold with $\left.\omega\right|_{\pi_{2} M}=0$ must have infinite fundamental group.

Again, let $M$ be any space. Suppose $\hat{\alpha} \in \pi_{1}\left(M^{M}, 1_{M}\right)$ with $\quad \mathrm{ev}_{\#}(\hat{\alpha})=\alpha \in$ $G(M)$. Recall that $\hat{\alpha}$ has a corresponding Wang derivation $\lambda_{\hat{\alpha}}$. Let $\pi^{\prime}$ denote the quotient $\pi /\langle\alpha\rangle$ and $p: \pi \rightarrow \pi^{\prime}$ the quotient homomorphism.

Theorem 4.4. Suppose $\omega \in H^{2}(M)$ satisfies $\left.\omega\right|_{\pi_{2} M}=0$, and that $f^{*}\left(\omega_{\pi}\right)=\omega$ as in Lemma 4.2. With the above notation, $\lambda_{\hat{\alpha}}(\omega)=0$ if and only if there exists $\omega_{\pi^{\prime}} \in H^{2}\left(K\left(\pi^{\prime}, 1\right)\right)$ with $p^{*} \omega_{\pi^{\prime}}=\omega_{\pi}$.

Proof. Because $\alpha$ is in the center $Z \pi$ of $\pi$, there is a homomorphism $\mathbb{Z} \times \pi \rightarrow$ $\pi$ given by $(n, g) \rightarrow \alpha^{n} g$. Further, this homomorphism is realized by a map 
of spaces $A_{\pi}: S^{1} \times K(\pi, 1) \rightarrow K(\pi, 1)$ and the following diagram homotopy commutes:

$$
\begin{array}{ccc}
M & \stackrel{f}{\longrightarrow} & K(\pi, 1) \\
A \uparrow & & \uparrow A_{\pi} \\
S^{1} \times M & \stackrel{1 \times f}{\longrightarrow} & S^{1} \times K(\pi, 1) .
\end{array}
$$

We now may calculate

$$
\begin{aligned}
A^{*} \omega & =A^{*} f^{*} \omega_{\pi} \quad \text { by Lemma } 4.2 \\
& =(1 \times f)^{*} A_{\pi}^{*} \omega_{\pi} \\
& =(1 \times f)^{*}\left(1 \times \omega_{\pi}+\bar{\sigma} \times \lambda_{\alpha}\left(\omega_{\pi}\right)\right)
\end{aligned}
$$

where $\lambda_{\alpha}$ is the Wang derivation associated to the clutching map $A_{\pi}$. Continuing,

$$
\begin{aligned}
A^{*} \omega & =1 \times f^{*} \omega_{\pi}+\dot{\bar{\sigma}} \times f^{*} \lambda_{\alpha}\left(\omega_{\pi}\right) \\
& =1 \times \omega+\bar{\sigma} \times f^{*} \lambda_{\alpha}\left(\omega_{\pi}\right) .
\end{aligned}
$$

Since $A^{*} \omega=1 \times \omega+\bar{\sigma} \times \lambda_{\hat{\alpha}}(\omega)$, we see that

$$
\lambda_{\hat{\alpha}}(\omega)=f^{*} \lambda_{\alpha}\left(\omega_{\pi}\right) .
$$

Then, because $f^{*}$ is an isomorphism on $H^{1}, \lambda_{\hat{\alpha}}(\omega)=0$ if and only if $\lambda_{\alpha}\left(\omega_{\pi}\right)=$ 0 .

If $\alpha$ has finite order, then $\pi$ and $\pi^{\prime}$ have isomorphic rational cohomology and, by Lemma 2.1, $\lambda_{\alpha}=0$. Thus, in this case, the theorem says that $\lambda_{\hat{\alpha}}(\omega)=0$ always.

In general, the element $\lambda_{\alpha}\left(\omega_{\pi}\right)=0$ may be analyzed by using $A_{\pi}$ to construct a fibration

$$
K(\pi, 1) \stackrel{i}{\rightarrow} E_{\pi} \rightarrow S^{2}
$$

with $i_{\#}=p: \pi \rightarrow \pi_{1} E_{\pi}=\pi^{\prime}$ and $\pi_{2} E_{\pi}=0$. By Theorem 4.1, the classifying map $g: E_{\pi} \rightarrow K\left(\pi^{\prime}, 1\right)$ induces an isomorphism $g^{*}: H^{2}\left(K\left(\pi^{\prime}, 1\right)\right) \rightarrow H^{2}\left(E_{\pi}\right)$ and the Wang sequence becomes

$$
\cdots \rightarrow H^{2}\left(K\left(\pi^{\prime}, 1\right)\right) \stackrel{p^{*}}{\rightarrow} H^{2}(K(\pi, 1)) \stackrel{\lambda_{a}}{\rightarrow} H^{1}(K(\pi, 1)) \rightarrow \cdots .
$$

Then, $\lambda_{\alpha}\left(\omega_{\pi}\right)=0$ if and only if there exists $\omega_{\pi^{\prime}}$ with $p^{*} \omega_{\pi^{\prime}}=\omega_{\pi}$.

To see how the above results may apply to c-symplectic spaces, we introduce the following notation: If a finitely generated group $\pi$ has $\sup \left\{k \mid H^{k}(\pi ; \mathbb{Q}) \neq\right.$ $0\}=N<\infty$, then we say $N$ is the $\mathbb{Q}$-dimension of $\pi$ and write $\operatorname{dim}_{\mathbb{Q}}(\pi)=N$. We say that $\alpha \in Z \pi$ is ( $\mathbb{Q}$-)dimension lowering if the quotient $\pi^{\prime}=\pi /\langle\alpha\rangle$ has $\operatorname{dim}_{\mathbb{Q}}\left(\pi^{\prime}\right)<\operatorname{dim}_{\mathbb{Q}}(\pi)$. If a group $\pi$ has an $\alpha \in Z \pi$ which is dimension lowering, then we say that $\pi$ is centrally dimension lowering, or is CDL.

Examples 4.5.

1. If $\pi$ is torsionfree nilpotent of rank $r$, then there is a refinement of the upper central series which displays a central $\mathbb{Z}$ and an extension $\mathbb{Z} \rightarrow \pi \rightarrow \pi^{\prime}$ with $\pi^{\prime}$ torsionfree nilpotent of rank $r-1$. Since the rank equals the dimension for these groups, such groups are CDL. (cf. $\left.\left[\mathrm{Op}_{1}\right]\right)$. 
2. More generally, if $M=K(\pi, 1)$ is any orientable (cohomology) manifold having a nontrivial $S^{1}$-action, then $\pi$ is CDL. This is true for the following reasons. It is known [C-R 3 ] that the orbit map $\alpha: S^{1} \rightarrow M$ induces an injection on fundamental groups, so there can be no fixed points. If an $S^{1}$-action has no fixed points, then it must act with finite isotropy - essentially because an infinite subgroup of $S^{1}$ is dense. It follows from the Leray spectral sequence that there is an isomorphism of rational cohomology $H^{*}\left(M S^{1}\right) \cong H^{*}\left(M / S^{1}\right)$ and that $\operatorname{dim} M / S^{1}=$ $\operatorname{dim} M-1$ [Br, IV, Theorem 3.8]. But then $M S^{1} \simeq K\left(\pi^{\prime}, 1\right)$ and $\operatorname{dim}_{\mathbb{Q}}\left(\pi^{\prime}\right)=\operatorname{dim} M-1<\operatorname{dim} M=\operatorname{dim}_{\mathbb{Q}}(\pi)$.

3. If $\pi^{\prime}$ has finite dimensional rational cohomology, then the product $\pi=$ $\mathbb{Z} \times \pi^{\prime}$ is CDL. For instance, $\pi^{\prime}$ could be the fundamental group of any compact $K(\pi, 1)$ such as a surface. A more interesting example of this is the fundamental group of the solvmanifold constructed in $\left[B-G_{2}\right.$, Example 1].

4. More generally, if $\pi^{\prime}$ has finite dimensional rational cohomology, then any class $\rho \in H^{2}\left(\pi^{\prime} ; \mathbb{Z}\right)$ constructs an extension $\mathbb{Z} \rightarrow \pi \rightarrow \pi^{\prime}$ with $\pi$ CDL.

One way to ensure the hypothesis $\left.\omega\right|_{\pi_{2} M}=0$, of Theorem 4.4, is satisfied is to take $M$ to be aspherical. Using notation as above, we have the following results.

Corollary 4.6. Let $(M, \omega)$ be a c-symplectic $2 n$-manifold of Lefschetz type, with $M$ a $K(\pi, 1)$. If $\hat{\alpha} \in \pi_{1}\left(M^{M}, 1_{M}\right)$ with $\mathrm{ev}_{\#}(\hat{\alpha})=\alpha$ dimension lowering, then $\langle\alpha\rangle \cap[\pi, \pi]=\{1\}$.

Proof. Suppose $\alpha^{s} \in\langle\alpha\rangle \cap[\pi, \pi]$ is nontrivial. Because $\alpha^{s} \in[\pi, \pi]$, the Hurewicz image of $\alpha$ is of finite order in $H_{1}(M ; \mathbb{Z})$ and the rational class $h(\alpha)$ vanishes. The Lefschetz type condition then implies that $\lambda_{\hat{\alpha}}(\omega)=0$. Using the fact that $\pi_{2} M=0$, Theorem 4.4 then provides $\omega_{\pi^{\prime}} \in H^{2}\left(K\left(\pi^{\prime}, 1\right)\right)$ with $p^{*} \omega_{\pi^{\prime}}=\omega_{\pi}$. The dimension lowering hypothesis entails $\operatorname{dim}_{\mathbb{Q}}\left(\pi^{\prime}\right)<\operatorname{dim}_{\mathbb{Q}}(\pi)$ and, consequently, $\omega_{\pi^{\prime}}^{n}=0$. This leads to the following contradiction:

$$
0 \neq \omega_{\pi}^{n}=\left(p^{*} \omega_{\pi^{\prime}}\right)^{n}=p^{*} \omega_{\pi^{\prime}}^{n}=0 .
$$

Recall that a $K(\pi, 1)$-manifold $M$ has a maximal toral action if there is an action of $T^{k}$ on $M$ such that the orbit map $a: T^{k} \rightarrow M$ induces an isomorphism $\pi_{1} T^{k} \cong Z \pi$. Many $K(\pi, 1)$-manifolds, including compact solvmanifolds for example, are known to have maximal toral actions (cf. [L-R]). In case $M$ has a maximal toral action, any element $\alpha \in Z \pi$ is dimension lowering. In this case then, Corollary 4.6 says that $Z \pi \cap[\pi, \pi]=\{1\}$.

Corollary 4.7. A nilmanifold $M$ of Lefschetz type is diffeomorphic to a torus.

Proof. $M=K(\pi, 1)$ with $\pi$ torsionfree nilpotent, $Z \pi \neq\{1\}$ and $Z \pi \cap$ $[\pi, \pi] \neq\{1\}$ unless $\pi$ is abelian (cf. [Wa, Theorem 1.3]). As in Example 4.5(1), there is a central extension $\mathbb{Z}=\langle\alpha\rangle \rightarrow \pi \rightarrow \pi^{\prime}$ with $\alpha$ dimension lowering. Without loss of generality, since this result only depends on the rational type of $\pi$, we may assume that the extension is rationally nontrivial. But then, considering the 5-term exact sequence in group homology (with $\mathbb{Q}$ coefficients) and identifying the connecting homomorphism with the nonzero transgression in the Lyndon-Hochschild-Serre spectral sequence, we see that 
$H_{1}(\pi ; \mathbb{Q}) \cong H_{1}\left(\pi^{\prime} ; \mathbb{Q}\right)$. Hence, $\alpha^{s} \in[\pi, \pi]$ for some $s$. This contradicts Corollary 4.6; hence, $\pi$ is free abelian (since it is finitely generated). A theorem of Mostow [Mo] characterizes nilmanifolds (or more generally, solvmanifolds) up to diffeomorphism by their fundamental groups. Therefore, $M$ is a torus.

Remark 4.8. This is Theorem B. Compare the Lie theoretic proof of $\left[\mathrm{B}-\mathrm{G}_{1}\right]$, the circle action proof of $\left[\mathrm{McD}_{2}\right]$ (which is in the same spirit as the one above) and the minimal model proof of [L-O].

Now suppose we have an $S^{1}$-action $A: S^{1} \times M \rightarrow M$ on a c-symplectic manifold $(M, \omega)$ with orbit map $\alpha: S^{1} \rightarrow M$ and fundamental group quotient homomorphism $p: \pi=\pi_{1} M \rightarrow \pi /\langle\alpha\rangle=\pi^{\prime}$. Because homotopy classes of maps of spaces into $K(G, 1)$ 's are classified at the fundamental group level, we have a homotopy commutative diagram

$$
\begin{array}{ccc}
M & \stackrel{f}{\rightarrow} & K(\pi, 1) \\
i \downarrow & & \downarrow \\
M S^{1} & \stackrel{f^{\prime}}{\rightarrow} & K\left(\pi^{\prime}, 1\right)
\end{array}
$$

which induces a commutative diagram

$$
\begin{array}{ccc}
H^{*}(M) & \stackrel{f^{*}}{\leftarrow} & H^{*}(K(\pi, 1)) \\
i^{*} \uparrow & & \uparrow p^{*} \\
H^{*}\left(M S^{1}\right) & \stackrel{f^{\prime}}{\leftarrow} & H^{*}\left(K\left(\pi^{\prime}, 1\right)\right) .
\end{array}
$$

As in Example 4.5.2 above, if $\alpha$ is nontrivial, then the action has no fixed points and we have the rational cohomology isomorphism $H^{*}\left(M S^{1}\right) \cong H^{*}\left(M / S^{1}\right)$. Therefore, in this situation, $H^{*}\left(M S^{1}\right)$ may be replaced in the diagram by $H^{*}\left(M / S^{1}\right)$. It is a beautiful theorem of Browder and Hsiang that, in fact, this replacement can be made quite generally:

Theorem 4.9 [B-H]. Suppose $M^{N}$ is a manifold and $f: M \rightarrow K(\Gamma, 1)$ is a map which induces a surjection $f_{\#}: \pi_{1} M \rightarrow \Gamma$. Suppose $G$ is a compact, connected Lie group and $A: G \times M \rightarrow M$ is a smooth, effective action on $M$ with orbit map $a: G \rightarrow M$. Denote the quotient $\Gamma / f_{\#} a_{\#}\left(\pi_{1} G\right)$ by $\Gamma^{\prime}$. Then there is a commutative diagram

$$
\begin{array}{ccc}
H^{*}(M) & \stackrel{f^{*}}{\leftarrow} & H^{*}(K(\Gamma, 1)) \\
q^{*} \uparrow & & \uparrow p^{*} \\
H^{*}(M / G) & \stackrel{g}{\longleftarrow} & H^{*}\left(K\left(\Gamma^{\prime}, 1\right)\right) .
\end{array}
$$

where $q: M \rightarrow M / G$ is the orbit space quotient.

Remark 4.10. As noted in [B-H], the homomorphism $g$ may not be induced by a map of spaces. Also, according to the remark at the end of $\S 1$ of [B-H], the theorem in fact holds for topological actions which can be equivariantly embedded in a smooth manifold such that the embedding has a smooth regular 
neighborhood. Finally, when we are concerned with not necessarily smooth actions, the results of [Ber] may be used in place of Theorem 4.9.

To apply this result in our context, we introduce the following definition:

Definition 4.11. Say that $M^{N}$ is a $\kappa$-manifold if there is a map $f: M \rightarrow$ $K(\Gamma, 1)$ that satisfies $f_{\#}: \pi_{1} M \rightarrow \Gamma$ surjective and $f^{*}: H^{N}(K(\Gamma, 1) ; \mathbb{Q}) \rightarrow$ $H^{N}(M ; \mathbb{Q})$ surjective.

Theorem 4.12. Suppose $M^{N}$ is a $\kappa$-manifold. If $\alpha: S^{1} \rightarrow M$ is the orbit map of a nontrivial $S^{1}$-action on $M$, then $\alpha$ is of infinite order in $G(M) \subseteq \pi_{1} M$.

Proof. If $\alpha$ is of finite order, then $H^{*}(\Gamma) \cong H^{*}\left(\Gamma^{\prime}\right)$ and the diagram from the Browder-Hsiang theorem above becomes

$$
\begin{array}{cll}
H^{*}(M) & f^{*} & H^{*}(K(\Gamma, 1)) \\
q^{*} \uparrow & \swarrow g \\
H^{*}\left(M / S^{1}\right) &
\end{array}
$$

The hypothesis on $M$ implies that $H^{N}(K(\Gamma, 1)) \rightarrow H^{N}(M)$ is surjective. However, the diagram shows that this homomorphism factors through $H^{N}\left(M / S^{1}\right)$ and this group is trivial since $\operatorname{dim}\left(M / S^{1}\right)=N-1$ (cf. [Br, IV, Theorem 3.8], although it is known that this result holds for topological actions as well).

Corollary 4.13. Any $S^{1}$-action on a $\kappa$-manifold $M$ has no fixed points. Proof. A fixed point would imply the orbit map $S^{1} \rightarrow M$ is inessential.

Corollary 4.14. If $M$ is a $\kappa$-manifold with $Z \pi$ finite, then $M$ has no nontrivial circle actions.

Proof. This follows since $G(M) \subseteq Z \pi$.

The following theorem provides the link to c-symplectic manifolds.

Theorem 4.15. If $\left(M^{2 n}, \omega\right)$ is a c-symplectic manifold with $\left.\omega\right|_{\pi_{2} M}=0$, then $M$ is a $\kappa$-manifold.

Proof. The classifying map $f: M \rightarrow K(\pi, 1)$ induces the identity on $\pi=$ $\pi_{1} M$. From Lemma 4.2, there is an $\omega_{\pi} \in H^{2}(K(\pi, 1))$ such that $f^{*} \omega_{\pi}=\omega$. Because $M$ is c-symplectic, $f^{*} \omega_{\pi}^{n}=\omega^{n} \neq 0$. Hence, $f^{*}$ is surjective on $H^{2 n}$.

We have the immediate consequences:

Theorem 4.16. Any $S^{1}$-action on a c-symplectic manifold $(M, \omega)$ with $\left.\omega\right|_{\pi_{2} M}=$ 0 has no fixed points and, therefore, is not c-Hamiltonian.

Proof. Combine Theorem 4.15 and Corollary 4.13. The second assertion follows from Corollary 3.7.

Corollary 4.17. If $(M, \omega)$ is a c-symplectic manifold with $\left.\omega\right|_{\pi_{2} M}=0$ and $Z \pi$ finite, then $M$ has no nontrivial circle actions.

Proof. Combine Theorem 4.15 and Corollary 4.14.

Remark 4.18. Clearly, Ono's result Theorem $C$ follows from Theorem 4.16 by the identification $\lambda_{\hat{\alpha}}(\omega)=[i(\underline{X}) \omega]$ in the case of a symplectic action on a symplectic manifold.

By combining Theorem 4.16 with Theorem 3.11 , we obtain the following result: 
Corollary 4.19. If $(M, \omega)$ is a symplectic manifold with $\left.\omega\right|_{\pi_{2} M}=0$ and $c_{1}(M)$ $=r \cdot \omega$, then there are no nontrivial symplectic circle actions on $M$.

Remark 4.20. The same lines of argument as above show that any c-symplectic $(M, \omega)$ with $\left.\omega\right|_{\pi_{2} M}=0$ has no nontrivial actions by semi-simple Lie groups. This follows since the fundamental groups of these Lie groups are finite and this gives (with notation as in Theorem 4.9) $H^{*}(K(\pi, 1)) \cong H^{*}\left(K\left(\pi^{\prime}, 1\right)\right.$ ).

Finally, we mention Gompf's Theorem [Gom] that every finitely presented group arises as the fundamental group of a symplectic manifold (which even may be taken to have dimension 4 ). Thus, the results of this section may be viewed as restrictions on the fundamental groups of c-symplectic manifolds under the hypothesis $\left.\omega\right|_{\pi_{2} M}=0$ and its relation to elements of $\pi_{1}\left(M^{M}, 1_{M}\right)$ (including the adjoints of $S^{1}$-actions).

\section{EXTENSIONS TO TORI}

The results of the first four sections also hold in a more general homotopical context which is the analogue of a torus action. Because the notation is rather complicated, we have chosen to present our results for circle actions fully and to merely outline the extensions here. Note, however, that this section also contains new results which apply to the circle actions we have previously considered. The reasons for discussing these here will be apparent.

Suppose $(M, \omega)$ is a c-symplectic manifold. Let $\mathscr{A}=\left\langle\hat{\alpha}_{1}, \ldots, \hat{\alpha}_{k}\right\rangle \subseteq$ $\pi_{1}\left(M^{M}, 1_{M}\right)$ be the subgroup generated by $\hat{\alpha}_{1}, \ldots, \hat{\alpha}_{k}$. Each $\hat{\alpha}_{i} \in \pi_{1}\left(M^{M}, 1_{M}\right)$ provides an element of $\pi_{2} B$ aut $(M)$ and these may be assembled to form a map $S^{2} \vee \cdots \vee S^{2} \rightarrow B$ aut $(M)$. The pullback of the universal fibration then gives a 'generalized Wang fibration'

$$
M \stackrel{i}{\rightarrow} E \stackrel{p}{\rightarrow} S^{2} \vee \cdots \vee S^{2} .
$$

Associated to this fibration is a generalized Wang sequence [Sp, p. 455],

$$
\cdots \rightarrow H^{q}(E) \stackrel{i^{*}}{\rightarrow} H^{q}(M) \stackrel{\mathrm{n}_{\hat{\alpha}_{i}}}{\longrightarrow} \Pi H^{q-1}(M) \rightarrow \cdots
$$

where we have identified the finite direct sum of $H^{q-1}(M)$ 's with the direct product. The following result is immediate from the exactness of the generalized Wang sequence and $\omega^{n} \neq 0$ :

\section{Proposition 5.1.}

1. $\lambda_{\hat{\alpha}_{i}}(\omega)=0$ for all $i=1, \ldots, k$ if and only if there exists $\bar{\omega} \in H^{2}(E)$ with $i^{*} \bar{\omega}=\omega$.

2. If $\lambda_{\hat{\alpha}_{i}}(\omega)=0$ for all $i=1, \ldots, k$, the fibre number $\Phi(p)$ is nonzero.

Say that $\mathscr{A}$ is $c$-Hamiltonian if $\lambda_{\hat{\alpha}_{i}}(\omega)=0$ for $i=1, \ldots, k$. It is a fact that $\lambda_{\alpha+\beta}=\lambda_{\alpha}+\lambda_{\beta}\left[\mathrm{Go}_{2}\right.$ ], so this definition is equivalent to saying $\lambda_{\gamma}(\omega)=0$ for all $\gamma \in \mathscr{A}$. Proposition 5.1 then says that if $\mathscr{A}$ is c-Hamiltonian, then $\Phi(p) \neq 0$. Now, from another point of view, the $\hat{\alpha}_{i}$ may be assembled into a map $S^{1} \vee \cdots \vee S^{1} \rightarrow\left(M^{M}, 1_{M}\right)$ which has the following extension:

$$
\begin{array}{ccc}
S^{1} \vee \cdots \vee S^{1} & \rightarrow & \left(M^{M}, 1_{M}\right) \\
\downarrow & \uparrow & \uparrow \\
S^{1} \times \cdots \times S^{1} & \rightarrow & \left(M^{M}, 1_{M}\right) \times \cdots \times\left(M^{M}, 1_{M}\right) .
\end{array}
$$


Therefore, given $\hat{\alpha}_{1}, \ldots, \hat{\alpha}_{k} \in \pi_{1}\left(M^{M}, 1_{M}\right)$, we obtain a map $\hat{\alpha}: T^{k} \rightarrow$ $\left(M^{M}, 1_{M}\right)$ which realizes $\hat{\alpha}_{i}$ on the $i$ th copy of $S^{1}$ and gives a surjection $\theta: \pi_{1}\left(T^{k}\right) \cong H^{1}\left(T^{k} ; \mathbb{Z}\right) \cong \mathbb{Z}^{k} \rightarrow \mathscr{A}$ (determined by $\left.\theta\left(\bar{\sigma}_{i}\right)=\hat{\alpha}_{i}\right)$. The adjoint map is then

$$
T^{k} \times M \stackrel{A}{\longrightarrow} M
$$

and the induced map on cohomology gives

$$
A^{*} \omega=1 \times \omega+\bar{\sigma}_{1} \times \lambda_{\hat{\alpha}_{1}}(\omega)+\cdots+\bar{\sigma}_{k} \times \lambda_{\hat{\alpha}_{k}}(\omega)+a^{*}(\omega) \times 1
$$

where $a: T^{k} \rightarrow M$ is the evaluation map and $\bar{\sigma}_{i} \in H^{1}\left(S_{i}^{1}\right)$ is a generator for the cohomology of the $i$ th circle. There is a linear map of vector spaces $\psi: H^{1}\left(T^{k}\right) \rightarrow H^{1}(M)$ determined by $\psi\left(\bar{\sigma}_{i}\right)=\lambda_{\hat{\alpha}_{i}}(\omega)$. By analogy with $\left[\mathrm{Gi}_{1}\right.$, Definition 2.1], we say that $\mathscr{A}$ is cohomologically free if $\psi$ is a monomorphism. This definition agrees with Ginzburg's when $T^{k}$ acts symplectically on a symplectic manifold $(M, \omega)$. To see this, we simply note that Lemma 2.4 extends as in Remark 2.5 to give a formula (at the level of forms)

$$
A^{*} \omega=p_{2}^{*} \omega+p_{1}^{*} \nu_{1} \wedge p_{2}^{*} i\left(\underline{X}_{1}\right) \omega+\cdots+p_{1}^{*} \nu_{k} \wedge p_{2}^{*} i\left(\underline{X}_{k}\right) \omega+a^{*}(\omega),
$$

where $\underline{X}_{i}$ is the fundamental vector field associated to the action of the $i$ th circle, $\nu_{i}$ is the volume form dual to $X_{i}$ and $a^{*}$ is induced from the orbit map $a: T^{k} \rightarrow M$. Clearly we again have $\lambda_{\hat{\alpha}_{i}}(\omega)=\left[i\left(\underline{X}_{i}\right) \omega\right]$ and the usual notion of a Hamiltonian action (i.e., $\left[i\left(\underline{X}_{i}\right) \omega\right]=0$ for all $i$ ) agrees with our definition above. Of course, the same relationship as before holds between the Wang fibration and the Borel fibration (e.g., $H^{2}(E) \cong H^{2}\left(M T^{k}\right)$ ), so Proposition 5.1 provides the following analogue of Corollary 3.7.

Theorem 5.2. A c-Hamiltonian $T^{k}$-action on a c-symplectic manifold has nonempty fixed point set.

V. Ginzburg has pointed out that Theorem 5.2 may be reduced to Corollary 3.7 in the smooth case (cf. [Gi-W]). Namely, since only a finite number of subgroups of $T^{k}$ appear as isotropy groups of points of $M$, there exists an $S^{1} \subset T^{k}$ which intersects each such isotropy group in a discrete subgroup. For this $S^{1}$, the fixed point sets of $T^{k}$ and $S^{1}$ agree. This reduces Theorem 5.2 to Corollary 3.7.

As we have said, it is not our intention here to restate every result of the previous sections for toral actions (or, more generally, $\mathscr{A}$ ). Instead, we will focus on a splitting theorem of Conner-Raymond type and its relationship with Ginzburg's splitting of symplectic toral actions. With this in mind, consider a subgroup $\mathscr{A}$ as above and the associated linear map $\psi: H^{1}\left(T^{k}\right) \rightarrow H^{1}(M)$. Define $\bar{\psi}$ to be the composition of $\psi$ with the coefficient homomorphism on cohomology induced by $\mathbb{Z} \rightarrow \mathbb{Q}$

$$
\bar{\psi}: H^{1}\left(T^{k} ; \mathbb{Z}\right) \rightarrow H^{1}\left(T^{k}\right) \rightarrow H^{1}(M) .
$$

Lemma 5.3. Let $\psi: \mathbb{Z}^{k} \rightarrow V$ be an abelian group homomorphism to a rational vector space $V$ and let $I=\langle\operatorname{Im} \psi\rangle$ denote the subspace of $V$ generated by $\operatorname{Im} \psi$. If $\operatorname{dim}(I)=r$, then there exists an isomorphism $\mathbb{Z}^{r} \times \mathbb{Z}^{k-r} \cong \mathbb{Z}^{k}$ with $\left.\psi\right|_{\mathbb{Z}^{r}}$ injective and $\psi\left(\mathbb{Z}^{k-r}\right)=0$. 
Proof. Consider the following factorization of $\psi$

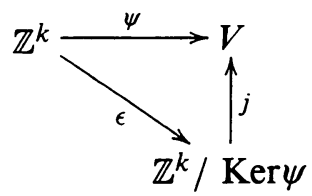

where $\epsilon$ is the quotient $\mathbb{Z}^{k} \rightarrow \mathbb{Z}^{k} / \operatorname{Ker} \psi \cong \mathbb{Z}^{r} \oplus F$ ( $F$ finite) and $j$ is the evident injection on the quotient. Note that the injectivity of $j$ implies the rank of $\mathbb{Z}^{k} / \operatorname{Ker} \psi$ is $r$.

There is a partial splitting $s: \mathbb{Z}^{r} \rightarrow \mathbb{Z}^{k}$ with $\epsilon s=1$ and $\epsilon^{-1}(F) \cap s\left(\mathbb{Z}^{r}\right)=\{0\}$. Further, if $\epsilon(z)=z_{1}+f$, then $\epsilon\left(z-s\left(z_{1}\right)\right)=f$ and $z-s\left(z_{1}\right) \in \epsilon^{-1}(F)$. Hence, $s\left(\mathbb{Z}^{r}\right) \times \epsilon^{-1}(F) \cong \mathbb{Z}^{k}$. Now we identify $s\left(\mathbb{Z}^{r}\right)$ with $\mathbb{Z}^{r}$ and note that $\epsilon^{-1}(F)$ must be free abelian of complementary rank $k-r$. Thus we write $\mathbb{Z}^{r} \times \mathbb{Z}^{k-r} \cong \mathbb{Z}^{k}$. Since $\epsilon$ is injective on $\mathbb{Z}^{r}$ and $j$ is injective, then $\psi=j \epsilon$ is injective on $\mathbb{Z}^{r}$ as well. Also, $\psi\left(\mathbb{Z}^{k-r}\right)=j \epsilon\left(\mathbb{Z}^{k-r}\right)=j(F)=0$ since $j(F)$ is finite and $V$ has no torsion.

The decomposition above clearly extends to a decomposition $\mathbb{R}^{r} \times \mathbb{R}^{k-r} \cong \mathbb{R}^{k}$ by extending coefficients. Taking quotients, we obtain

$$
T^{r} \times T^{k-r} \cong \mathbb{R}^{r} / \mathbb{Z}^{r} \times \mathbb{R}^{k-r} / \mathbb{Z}^{k-r} \cong \mathbb{R}^{r} \times \mathbb{R}^{k-r} / \mathbb{Z}^{r} \times \mathbb{Z}^{k-r} \cong \mathbb{R}^{k} / \mathbb{Z}^{k} \cong T^{k}
$$

with the induced map on fundamental groups (and hence on first cohomology) being the splitting $\mathbb{Z}^{r} \times \mathbb{Z}^{k-r} \cong \mathbb{Z}^{k}$. Let us now apply this to the map $\bar{\psi}$ : $H^{1}\left(T^{k} ; \mathbb{Z}\right) \rightarrow H^{1}\left(T^{k}\right) \rightarrow H^{1}(M)$. First we note

Lemma 5.4. The following diagram commutes:

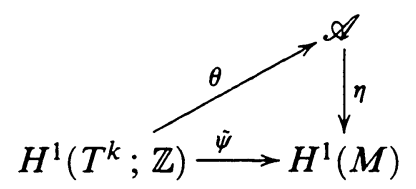

where $\eta\left(\hat{\alpha}_{i}\right)=\lambda_{\hat{\alpha}_{i}}(\omega)$.

Proof. The result is trivially true if we show $\eta$ is well defined. Since $\theta$ is a surjection, this will follow if $\operatorname{Ker} \theta \subseteq \operatorname{Ker} \bar{\psi}$. Suppose $z=\sum c_{i} \bar{\sigma}_{i} \in \operatorname{Ker} \theta$. Then $\theta(z)=\sum c_{i} \hat{\alpha}_{i}=0$ and $\bar{\psi}(z)=\sum c_{i} \lambda_{\hat{\alpha}_{i}}(\omega)=\lambda_{\Sigma c_{i} \hat{\alpha}_{i}}(\omega)=0$ as well.

Now $\theta: \mathbb{Z}^{k} \rightarrow \mathscr{A}$ is a surjection, so the splitting $\mathbb{Z}^{k} \cong \mathbb{Z}^{r} \times \mathbb{Z}^{k-r}$ induces a splitting $\mathscr{A} \cong \mathscr{A}_{r} \times \mathscr{A}_{k-r}$ with $\theta\left(\mathbb{Z}^{r}\right)=\mathscr{A}_{r}$ and $\theta\left(\mathbb{Z}^{k-r}\right)=\mathscr{A}_{k-r}$. Lemmas 5.3, 5.4 and the properties of $\bar{\psi}$ immediately imply

Theorem 5.5. Given $\mathscr{A} \subseteq \pi_{1}\left(M^{M}, 1_{M}\right)$ as above, there is a splitting $\mathscr{A}_{r} \times \mathscr{A}_{k-r} \cong$ $\mathscr{A}$ induced by $T^{r} \times T^{k-r} \cong T^{k}$ for which $\mathscr{A}_{r}$ is cohomologically free and $\mathscr{A}_{k-r}$ is c-Hamiltonian.

When $A: T^{k} \times M \rightarrow M$ is an action, then we say that, with respect to the splitting $T^{r} \times T^{k-r} \cong T^{k}$, the restricted action of $T^{r}$ is cohomologically free and that of $T^{k-r}$ is c-Hamiltonian. Thus we have

Corollary 5.6 (cf. [ $\mathrm{Gi}_{1}$, Proposition 4.2]). Any action of a torus $T^{k}$ on a csymplectic manifold $M$ splits as a cohomologically free action $T^{r} \times M \rightarrow M$ and a $c$-Hamiltonian action $T^{k-r} \times M \rightarrow M$ with $T^{r} \times T^{k-r} \cong T^{k}$. 
Remark 5.7. The reader should compare this result to the Reduction Theorem of [C-R 1 p. 184].

Now suppose $(M, \omega)$ is c-symplectic of Lefschetz type and

$$
\mathscr{A} \subseteq \pi_{1}\left(M^{M}, 1_{M}\right)
$$

is cohomologically free. Say that $\mathscr{A}$ is homologically injective if the composition

$$
T^{k} \stackrel{\hat{\alpha}}{\rightarrow}\left(M^{M}, 1_{M}\right) \stackrel{\text { ev }}{\rightarrow} M
$$

induces an injection $\mathbb{Z}^{k} \cong H_{1}\left(T^{k} ; \mathbb{Z}\right) \rightarrow H_{1}(M ; \mathbb{Z})$. Of course, this homomorphism factors through $\mathscr{A}$ by

$$
H_{1}\left(T^{k} ; \mathbb{Z}\right) \cong \pi_{1}\left(T^{k}\right) \rightarrow \mathscr{A} \stackrel{\mathrm{ev}^{*}}{\rightarrow} \pi_{1}(M) \stackrel{h}{\rightarrow} H_{1}(M ; \mathbb{Z})
$$

so the surjection $\mathbb{Z}^{k} \rightarrow \mathscr{A}$ is also an injection and hence an isomorphism. This notion of homological injectivity extends the usual notion for the action of a torus $T^{k}$ on $M$. That is, an action of $T^{k}$ on $M$ is homologically injective if the orbit map $T^{k} \rightarrow M$ induces an injection $H_{1}\left(T^{k} ; \mathbb{Z}\right) \rightarrow H_{1}(M ; \mathbb{Z})$. These actions have been studied extensively (cf. $\left[\mathrm{C}-\mathrm{R}_{2}, \mathrm{C}-\mathrm{R}_{3}\right.$ and Sa]). The main structural theorem is

\section{Theorem 5.8.}

1. $\left[\mathrm{Go}_{5}, \mathrm{Op}_{2}\right]$ If $\mathscr{A}$ is homologically injective of rank $r$, then there exists a finite abelian cover $\tilde{M} \rightarrow M$ with $\tilde{M} \simeq T^{r} \times Y$.

2. [C- $\mathrm{R}_{3}$ ] If an action of $T^{r}$ on $M$ is homologically injective, then $\left(T^{r}, M\right)$ is equivariantly homeomorphic to $\left(T^{r}, T^{r} \times{ }_{F} Y\right)$ where $F \subseteq T^{r}$ is a finite subgroup acting diagonally on $T^{r} \times Y$ and freely by translations on $T^{r}$. Further, the action of $T^{r}$ on $T^{r} \times Y$ is by translations on the first factor. Equivalently, we may say that $M$ fibres equivariantly over $T^{r} / F$ with fibre $Y$ and structure group $F$.

Our purpose here is to prove the fundamental

Theorem 5.9. If $(M, \omega)$ is a c-symplectic manifold of Lefschetz type, then $\mathscr{A}$ is cohomologically free if and only if $\mathscr{A}$ is homologically injective.

Proof. Suppose $\mathscr{A}$ is cohomologically free. Then $\psi: H^{1}\left(T^{k}\right) \cong \mathbb{Q}^{k} \rightarrow H^{1}(M)$ is injective. Choose a splitting $s: H^{1}(M) \rightarrow H^{1}\left(T^{k}\right)$ with $s \psi=1$. Also, denote by $P D: H_{1}(M) \cong H^{2 n-1}(M)$ the Poincare duality isomorphism and by $\phi: H^{1}(M) \cong H^{2 n-1}(M)$ the homomorphism $n$-times multiplication by $\omega^{n-1} ; \phi(u)=n u \omega^{n-1}$. Note that this is an isomorphism by the Lefschetz condition and the fact that we are dealing with rational vector spaces. Consider the composition

$$
H_{1}\left(T^{k}\right) \stackrel{\alpha_{*}}{\rightarrow} H_{1}(M) \stackrel{P D}{\rightarrow} H^{2 n-1}(M) \stackrel{\phi^{-1}}{\rightarrow} H^{1}(M) \stackrel{s}{\rightarrow} H^{1}\left(T^{k}\right) .
$$

Recalling from Lemma 3.1 that $h(\alpha)$ is Poincare dual to $n \lambda_{\hat{\alpha}}(\omega) \omega^{n-1}$, we obtain

$$
\begin{aligned}
s \phi^{-1} P D \alpha_{*}\left(\sigma_{i}\right) & =s \phi^{-1} P D h\left(\alpha_{i}\right)=s \phi^{-1} n \lambda_{\hat{\alpha}_{i}}(\omega) \omega^{n-1} \\
& =s \lambda_{\hat{\alpha}_{i}}(\omega)=s \psi\left(\bar{\sigma}_{i}\right)=\bar{\sigma}_{i} .
\end{aligned}
$$


Since the composition is an isomorphism (carrying generators to their duals), $\alpha_{*}$ is an injection. Then, since $H_{1}\left(T^{k} ; \mathbb{Z}\right)$ is torsionfree, $\alpha_{*}: H_{1}\left(T^{k} ; \mathbb{Z}\right) \rightarrow$ $H_{1}(M ; \mathbb{Z})$ is injective as well. Thus $\mathscr{A}$ is homologically injective.

Now suppose $\mathscr{A}$ is homologically injective. Since $H_{1}\left(T^{k} ; \mathbb{Z}\right)$ is torsionfree, $\alpha_{*}: H_{1}\left(T^{k}\right) \rightarrow H_{1}(M)$ is injective as well. Choose a splitting $t: H_{1}(M) \rightarrow$ $H_{1}\left(T^{k}\right)$ with $t \alpha_{*}=1$. Computing as above, we have $t P D^{-1} \phi \psi\left(\bar{\sigma}_{i}\right)=\sigma_{i}$ and so $\psi$ is an injection. Hence, $\mathscr{A}$ is cohomologically free.

Remark 5.10. Note that in the proof of the 'if' statement we only used the definition of $\phi$ and not the existence of an inverse $\phi^{-1}$. Therefore, even without the assumption of Lefschetz type, homologically injective implies cohomologically free.

We have the following extension of Theorem 3.8. See [ $\mathrm{Gi}_{1}$, Proposition 2.2] for the 'only if' part as well. Note that the proof of the 'if' part below makes no use of the Lefschetz type assumption and therefore holds for any c-symplectic manifold.

Theorem 5.11. Suppose $(M, \omega)$ is a c-symplectic manifold of Lefschetz type. Then $T^{k}$ acts cohomologically freely on $M$ if and only if all isotropy groups are finite.

Proof. First, assume the action is cohomologically free and suppose some isotropy group $T_{p}$ is infinite. Then $H_{1}\left(T^{k} ; \mathbb{Z}\right) \rightarrow H_{1}\left(T^{k} / T_{p} ; \mathbb{Z}\right)$ reduces rank and the orbit map $T^{k} \rightarrow M$ induces $H_{1}\left(T^{k} ; \mathbb{Z}\right) \rightarrow H_{1}\left(T^{k} / T_{p} ; \mathbb{Z}\right) \rightarrow H_{1}(M ; \mathbb{Z})$ which cannot be injective and this contradicts Theorem 5.9.

Now suppose each isotropy group is finite. By Corollary 5.6 we may decompose the torus $T^{k}$ as $T^{k} \cong T^{r} \times T^{k-r}$ with the restricted action cohomologically free on $T^{r}$ and c-Hamiltonian on $T^{k-r}$. Now, a c-Hamiltonian action has a fixed point, so this is true for the $T^{k-r}$ action. But this contradicts finiteness of isotropy unless $k-r=0$. Thus, $T^{k}=T^{r}$ and the action is cohomologically free.

Remark 5.12. For c-symplectic manifolds of Lefschetz type, Theorem 5.11 identifies the cohomologically free versus c-Hamiltonian splitting of a torus action with the standard fixed point versus finite isotropy splitting of transformation group theory. This should prove useful in identifying cohomologically free (and hence, homologically injective) actions. Compare this with [C-R $\mathrm{R}_{1}$, Theorem 12.6] where a homologically Kähler version of Theorem 5.11 is proved. Conner and Raymond point out that their result is a topological version of Calabi's reduction conjecture for closed Kähler manifolds. Thus Theorem 5.11 is a version of this conjecture for c-symplectic manifolds of Lefschetz type.

Note that in Corollary 4.6 we showed that a dimension lowering element in the center $Z \pi$ of a Lefschetz type c-symplectic $K(\pi, 1)$ is homologically injective. In fact, the proof shows that $\mathscr{A}=\langle\alpha\rangle$ must be cohomologically free, i.e., $\lambda_{\alpha}(\omega) \neq 0$. A generalization of this is the following result:

Corollary 5.13. If $(M, \omega)$ is c-symplectic with $\left.\omega\right|_{\pi_{2} M}=0$, then any $T^{k}$-action on $M$ is cohomologically free. If $M$ also has Lefschetz type, then any $T^{k}$-action is homologically injective.

Proof. Any $T^{k}$-action may be decomposed as $T^{k} \cong T^{s} \times T^{k-s}$ where the fixed point set $F\left(T^{s}, M\right) \neq \phi$ and $T^{k-s}$ acts with finite isotropy. But the torus ver- 
sion of Theorem 4.12 says that no torus action on such an $M$ can have fixed points. Hence, $s=0, T^{k}=T^{k-s}$ and $T^{k}$ acts with finite isotropy. By Theorem 5.11 and the discussion directly preceding it, the action is cohomologically free.

Example 5.14. If $M=K(\pi, 1)$ is a solvmanifold, then there is a maximal toral action [L-R]. That is, for $Z \pi=\mathbb{Z}^{k}$, there is an action of $T^{k}$ on $M$ with orbit map inducing $\pi_{1} T^{k} \cong Z \pi$. If $M$ is c-symplectic of Lefschetz type, then this torus action must be homologically injective. Theorem 5.8 then says that $M=T^{k} \times_{F} Y$ where $F \subseteq T^{k}$ is finite. So, any compact solvmanifold of Lefschetz type has a finite abelian cover which splits off a torus of the same rank as $Z \pi$.

Corollary 5.15. Let $(M, \omega)$ have Lefschetz type. If an action of $T^{r}$ on $M$ is cohomologically free, then $H^{*}(M) \cong H^{*}\left(M / T^{r}\right) \otimes H^{*}\left(T^{r}\right)$ as algebras.

Proof. First note that, by Corollary 5.9, all isotropy groups are finite. A Leray spectral sequence argument then gives $H^{*}\left(M T^{r}\right) \cong H^{*}\left(M / T^{r}\right)$. Now consider one step back in the Borel fibration $T^{r} \stackrel{\alpha}{\rightarrow} M \rightarrow M T^{r}$ and note that Theorem 5.8 implies the action is homologically injective. This translates into a surjection $\alpha^{*}: H^{1}(M) \rightarrow H^{1}\left(T^{r}\right)$. Choose a vector space splitting $s: H^{1}\left(T^{r}\right) \rightarrow H^{1}(M)$ with $\alpha^{*} S=1_{H^{1}\left(T^{r}\right)}$. But $H^{*}\left(T^{r}\right)$ is freely generated as an algebra by its degree one elements, so in fact, $s$ is an algebra splitting. Thus, the Leray-Hirsch

Theorem may be strengthened to provide an algebra isomorphism $H^{*}(M) \stackrel{\text { Alg }}{\cong}$ $H^{*}\left(M / T^{r}\right) \otimes H^{*}\left(T^{r}\right)$.

Theorem 5.11 and Corollary 5.15 provide an answer to a special case of a conjecture of Halperin [Ha]. Define the toral rank of a space $X$ to be the largest $k$ for which a torus $T^{k}$ can act with finite isotropy on $X$. This is denoted $\mathrm{rk}_{0}(X)$. Halperin conjectured that

$$
\operatorname{dim} H^{*}(X ; \mathbb{Q}) \geq 2^{\text {rko }_{0}(X)}
$$

and showed that the conjecture was true for homogeneous spaces. Allday and Puppe [A-P] later proved the conjecture for homologically Kählerian spaces. The cohomological splitting of Corollary 5.15 and the equivalence of cohomological freeness with finite isotropy (under the Lefschetz type hypothesis) imply

Theorem 5.16. Halperin's conjecture is true for any c-symplectic manifold of Lefschetz type.

The cohomological splitting of Corollary 5.15 may be considerably enhanced into a quite restrictive topological structure result which follows from Theorem 5.8 and Theorem 5.9:

Corollary 5.17. Suppose $(M, \omega)$ has Lefschetz type.

1. If $\mathscr{A}$ is cohomologically free of rank $r$, then there is a finite abelian cover $\tilde{M} \simeq T^{r} \times Y$.

2. If an action of $T^{r}$ on $M$ is cohomologically free, then $\left(T^{r}, M\right)$ is equivariantly homeomorphic to $\left(T^{r}, T^{r} \times{ }_{F} Y\right)$ where $F \subseteq T^{r}$ is a finite subgroup acting diagonally on $T^{r} \times Y$ and freely by translations on $T^{r}$. Further, the action of $T^{r}$ on $T^{r} \times Y$ is by translations on the first factor.

Of course all these results serve as tools to detect the nonexistence of cohomologically free $\mathscr{A}$ or $T^{r}$-actions on a c-symplectic $(M, \omega)$ of Lefschetz 
type. We end by giving one more such result due to Sadowski [Sa]. Say that a Riemannian metric on a $T^{k}$-manifold $M$ is $T^{k}$-parallel if the action of $T^{k}$ is generated by parallel fields. Then, Sadowski's Theorem, restricted to the Lefschetz type case and recast in the present language is

Theorem 5.18 [Sa]. Suppose $(M, \omega)$ is a symplectic manifold of Lefschetz type with a smooth effective action of $T^{k}$. Then $M$ has a $T^{k}$ parallel metric if and only if the action is cohomologically free.

\section{ACKNOWLEDGEMENTS}

We would like to thank Michèle Audin, Bob Gompf and Viktor Ginzburg for sending their reprints. Also, Bob Gompf, Viktor Ginzburg, Dusa McDuff and Dan Gottlieb provided several illuminating e-mail messages which greatly improved this work. In particular, the discussion of the Calabi invariant in $\S 3$ owes its existence to the interest and expertise of Viktor Ginzburg.

\section{References}

[A-M] R. Abraham and J. Marsden, Foundations of mechanics, 2nd ed., Addison-Wesley, 1978.

[A-P] C. Allday and V. Puppe, Bounds on the torus rank, Lecture Notes in Math., vol. 1217, Springer, 1986, pp. 1-10.

[A-B] M. Atiyah and R. Bott, The moment map and equivariant cohomology, Topology 23 (1984), 1-28.

$\left[\mathrm{Au}_{1}\right] \quad$ M. Audin, The topology of torus actions on symplectic manifolds, Progress in Math., 93, Birkhäuser, 1991.

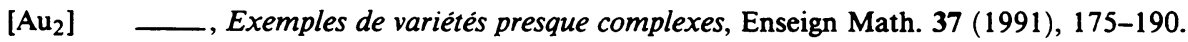

[Ban] A. Banyaga, Sur la structure du groupe des diffeomorphismes qui preservent une forme symplectique, Comment. Math. Helv. 53 (1978), 174-227.

[B-G $\mathrm{G}_{1}$ C. Benson and C. Gordon, Kähler and symplectic structures on nilmanifolds, Topology 27 (1988), 513-518.

[B-G $\left.\mathrm{G}_{2}\right] \quad$ Kähler structures on compact solvmanifolds, Proc. Amer. Math. Soc. 108 (1990), 971-980.

[Ber] I. Berstein, On covering spaces and Lie group actions, Contemp. Math. 37 (1985), 11-13.

[Br] G. Bredon, Introduction to compact transformation groups, Academic Press, 1972.

[B-H] W. Browder and W-C. Hsiang, G-actions and the fundamental group, Invent. Math. 65 (1982), 411-424.

[Cal] E. Calabi, On the group of automorphisms of a symplectic manifold, Problems in Analysis: Symposium in Honor of S. Bochner, Princeton Univ. Press, 1970, pp. 1-26.

[C-G] A. Casson and D. Gottlieb, Fibrations with compact fibres, Amer. J. Math. 99 (1977), 159-189.

[C-R $\mathrm{R}_{1}$ P. Conner and F. Raymond, Holomorphic Seifert fiberings, Lecture Notes in Math., vol. 299, 1972, pp. 124-204.

[C-R $\mathrm{R}_{2}$, Injective operations of the toral groups II, Lecture Notes in Math., vol. 299, Springer, 1972, pp. 109-123.

[C-R $\left.\mathbf{R}_{3}\right]$, Injective operations of the toral groups, Topology 10 (1971), 283-296.

[C-F-G] L. A. Cordero, M. Fernández and A. Gray, Symplectic manifolds with no Kähler structure, Topology 25 (1986), 375-380.

[Fl] A. Floer, Cuplength estimates on Lagrangian intersections, Comm. Pure Appl. Math. 42 (1989), 335-357.

[Fr] T. Frankel, Fixed points and torsion on Kähler manifolds, Ann. of Math. 70 (1959), 1-8. 
[Ge] H. Geiges, Symplectic structures on $T^{2}$-bundles over $T^{2}$, Duke Math. 67 (1992), 539-555.

[Gi ${ }_{1}$ V. Ginzburg, Some remarks on symplectic actions of compact groups, Math. Z. 210 (1992), 625-640.

[Gi $\left.{ }_{2}\right]-$ New generalizations of Poincaré's geometric theorem, Funct. Anal. Appl. 21 (1987), 100-106.

[Gi-W] V. Ginzburg and A. Weinstein, Lie-Poisson structure on some Poisson Lie groups, J. Amer. Math. Soc. 5 (1992), 445-453.

[Gom] R. Gompf, Some new symplectic 4-manifolds, preprint 1993.

[Go ${ }_{1}$ D. Gottlieb, A certain subgroup of the fundamental group, Amer. J. Math. 87 (1965), 840-856.

[Go2] D. Gottlieb, Applications of bundle map theory, Trans. Amer. Math. Soc. 171 (1972), 23-50.

[Go ] _ The trace of an action and the degree of a map, Trans. Amer. Math. Soc. 293 (1986), 381-410.

[Go4] D. Gottlieb, Lifting actions in fibrations, Lecture Notes in Math., vol. 657, Springer, 1977, pp. 217-254.

[Go5] - Splitting off tori and the evaluation subgroup, Israel J. Math. 66 (1989), 216-222.

[Go $]$ - Evaluation subgroups of homotopy groups, Amer. J. Math. 91 (1969), 729-756.

[Ha] S. Halperin, Rational homotopy and torus actions, Aspects of Topology, London Math. Soc. Lecture Notes, 93, 1985, pp. 1-20.

[L-R] K. B. Lee and F. Raymond, Maximal torus actions on solvmanifolds and double coset spaces, Internat. J. Math. 2 (1991), 67-76.

[L-O] G. Lupton and J. Oprea, Symplectic manifolds and formality, J. Pure Appl. Algebra 91 (1994), 193-207.

$\left[\mathrm{McD}_{1}\right]$ D. McDuff, Examples of simply-connected, symplectic non-Kählerian manifolds, J. Differential Geom. 20 (1984), 267-277.

$\left[\mathrm{McD}_{2}\right]$, The moment map for circle actions on symplectic manifolds, J. Geom. Phys. 5 (1988), 149-160.

$\left[\mathrm{McD}_{3}\right]$, Symplectic diffeomorphisms and the flux homomorphism, Invent. Math. 77 (1984), 353-366.

[Mo] G. Mostow, Factor spaces of solvable groups, Ann. of Math. 60 (1954), 1-27.

[Ol] P. Olver, Applications of Lie groups to differential equations, Graduate Texts in Math., vol. 107, Springer-Verlag, 1986.

$\left[\mathrm{On}_{1}\right] \mathrm{K}$. Ono, Equivariant projective imbedding theorem for symplectic manifolds, J. Fac. Sci. Univ. Tokyo IA Math. 35 (1988), 381-392.

[On $\left.\mathrm{On}_{2}\right]$, Obstruction to circle group actions preserving symplectic structure, Hokkaido Math. J. 21 (1992), 99-102.

[Op $\left.\mathrm{p}_{1}\right] \quad J$. Oprea, The category of nilmanifolds, Enseign. Math. 38 (1992), 27-40.

[Op $\left.{ }_{2}\right] \quad$ A homotopical Conner-Raymond Theorem and a question of Gottlieb, Canad. Bull. Math. 33 (1990), 219-229.

[Sa] M. Sadowski, Equivariant splittings associated with smooth toral actions, Lecture Notes in Math., vol. 1474, Springer, 1991, pp. 183-192.

[Sp] E. Spanier, Algebraic topology, McGraw-Hill, 1966.

[Th] W. Thurston, Some simple examples of symplectic manifolds, Proc. Amer. Math. Soc. 55 (1976), 467-468.

[Wa] R. Warfield, Nilpotent groups, Lecture Notes in Math., vol. 513, Springer-Verlag, 1976.

Department of Mathematics, Cleveland State University, Cleveland OH 44115

r12110vmcms . csuohio.edu

oprea@csvaxd.csuohio.edu 